University of Vermont

UVM ScholarWorks

2010

\title{
A Bound Vortex Surface Impingement Method for Adhered Dust Particle Removal
}

Nicholas Vachon

University of Vermont

Follow this and additional works at: https://scholarworks.uvm.edu/graddis

\section{Recommended Citation}

Vachon, Nicholas, "A Bound Vortex Surface Impingement Method for Adhered Dust Particle Removal" (2010). Graduate College Dissertations and Theses. 233.

https://scholarworks.uvm.edu/graddis/233

This Thesis is brought to you for free and open access by the Dissertations and Theses at UVM ScholarWorks. It has been accepted for inclusion in Graduate College Dissertations and Theses by an authorized administrator of UVM ScholarWorks. For more information, please contact scholarworks@uvm.edu. 


\title{
A BOUND VORTEX SURFACE IMPINGEMENT METHOD FOR ADHERED DUST PARTICLE REMOVAL
}

\author{
A Thesis Presented \\ by \\ Nicholas M. Vachon \\ to \\ The Faculty of the Graduate College \\ of \\ The University of Vermont \\ In Partial Fullfillment of the Requirements \\ for the Degree of Master of Science \\ Specializing in Mechanical Engineering
}

October, 2010 


\section{Abstract}

Methods of dust mitigation in Martian and Lunar environments are an increasingly active area of research within the fluid dynamic and aerospace community. Martian and Lunar environments produce electrically charged particles, which easily adhere to exposed surfaces. Adhered regolith particles can interfere with human comfort and mechanical functionality. In this work we investigate the potential to enhance particle removal through bound vortex surface impingement. A bound vortex flow condition is created using a specialized nozzle configuration where a combination of positive pressure inlets and a central negative pressure outlet are used to control flow dynamics. Using the techniques of computational fluid dynamic simulations and physical experiments, the effectiveness of vortex-induced flow conditions is evaluated. A parametric study is performed to explore bound vortex formation over a range of nozzle configurations and pressure conditions. Visualization of pathlines and measurement of shear stress under various geometric and pressure conditions provide insight into flow characteristics. It is found that an optimal range of key geometric and pressure parameters exist in the creation of bound vortex flow and such flow enhances particle transportation and removal. A subset of optimal computational configurations is recreated experimentally to support the existence of bound vortex flow and its positive impact on the removal of particles. 


\section{Acknowledgements}

The completion of this thesis could not have been achieved without the support of many individuals within the University of Vermont community and beyond. These individuals have provided the guidance, tools, and knowledge required to accomplish my goals.

First, I would like to express my deepest gratitude to my advisor, Prof. Darren Hitt, for his exceptional guidance, unwavering patience, and invaluable knowledge through

which I have gained a deepened understanding and respect for the engineering sciences.

I would like to thank the members of my thesis committee; Prof. Jeffrey Marshall, Prof. Mandar Dewoolkar, and Dr. William Louisos for their efforts in the successful completion of my thesis.

Finally, I would like to thank my wife and family for their support and understanding throughout my educational endeavors.

Funding for this work has been provided by NASA under Cooperative Agreement \#NNX08AZ07A 


\section{Table of Contents}

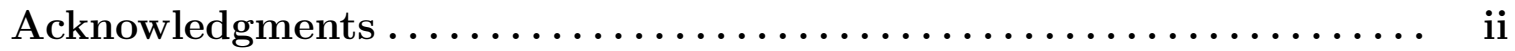

List of Tables................................... v

List of Figures............................. vi

Chapter 1 Introduction............................... 1

1.1 Existing Mitigation Strategies _. . . . . . . . . . . . . . 2

1.2 Surface Shear Stress . . . . . . . . . . . . . . . . . . . 3

1.3 Enhanced Flow Structures . . . . . . . . . . . . . . . . 5

1.4 Bound Vortex Flow . . . . . . . . . . . . . . . . . . . . . . 7

Chapter 2 Methodology $\ldots \ldots \ldots \ldots \ldots \ldots \ldots \ldots \ldots \ldots \ldots \ldots . \quad 9$

2.1 Nozzle Design . . . . . . . . . . . . . . . . . . . . . . . . . . . . 9

2.2 Computational Model . . . . . . . . . . . . . . . . . . . . . 11

2.3 Experimental Methods . . . . . . . . . . . . . . . . . . . 13

Chapter 3 Results................................ 19

3.1 Computational Results . . . . . . . . . . . . . . . . . . . . 19

3.2 Experimental Results . . . . . . . . . . . . . . . . . . . . . 23 
Chapter 4 Estimation of Micro-Particle Removal Efficiency ........ 48

4.1 Derivation of Critical Shear Condition for Particle Removal . . . . . . 49

4.2 Critical Shear Values for Micro-Particles . . . . . . . . . . . . . 50

Chapter 5 Conclusions $\ldots \ldots \ldots \ldots \ldots \ldots \ldots \ldots \ldots \ldots \ldots \ldots \ldots \ldots$

5.1 Future Work . . . . . . . . . . . . . . . . 58

References $\ldots \ldots \ldots \ldots \ldots \ldots \ldots \ldots \ldots \ldots \ldots \ldots \ldots \ldots \ldots \ldots, 60$

Appendix A Fluent Formulation $[3] \ldots \ldots \ldots \ldots \ldots \ldots \ldots \ldots \ldots, 64$

A.1 Navier-Stokes Equations . . . . . . . . . . . . . . 64

A.2 Realizable $k-\epsilon$ Turbulence Model . . . . . . . . . . . . . . . . . 65

A.3 Pressure-Based Solver . . . . . . . . . . . . . . . . 66

A.4 Discretization of the Continuity Equation . . . . . . . . . . . . 67

A.5 Pressure-Velocity Coupling . . . . . . . . . . . . . . . 67

A.6 Second-Order Upwind Scheme . . . . . . . . . . . . . . . . 68

A.7 Nozzle Design . . . . . . . . . . . . . . . . . . . 70 


\section{List of Tables}

3.1 Geometric nozzle parameters resulting in 12 nozzle configurations. . . 25

3.2 Seven pressure conditions applied to each nozzle. . . . . . . . . . . 26

3.3 Pressure conditions and corresponding Reynolds numbers are shown for a fixed pressure ratio and varying pressure magnitude. Reynolds numbers were calculated for the positive pressure jets based on a diameter of $1.5 \mathrm{~mm} . \ldots \ldots \ldots$. . . . . . . . . . . . . . 42

4.1 Critical shear stress is shown as a function of particle size. . . . . . . 52 


\section{List of Figures}

1.1 Lunar regolith simulant particles are nonuniform, sharp, and abrasive. Martian and Lunar dust particles can negatively impact human health and reduce the mechanical longevity of equipment. . . . . . . . .

2.1 A drawing depicting a basic nozzle geometry consisting of a single 5 $\mathrm{mm}$ central port and ten equally spaced $1.5 \mathrm{~mm}$ jets. The positive pressure jets are defined by a tangential and radial component. . . .

2.2 A computational grid comprised of tetrahedral elements is shown. Tetrahedral grid density increases within the domain as the impinged surface is approached. Boundary conditions are defined by the following color key: Blue - Pressure Outlet; Red - No-Slip Wall; Green - Positive Pressure Inlet; Yellow - Negative Pressure Outlet. . . . . . . . . . .

2.3 Average and peak shear stress on the impinged surface is shown for a constant geometry of $60^{\circ}$ tangential component and $15^{\circ}$ radial component. The inlet pressure is held at a constant $10 \mathrm{kPa}$ and the outlet pressure at a constant $-40 \mathrm{kPa}$. The value of peak and average shear stress remain constant at a grid resolution at or greater than 4000 elements per meter. . . . . . . . . . . . . . . . . 
2.4 Twelve nozzles rapid-prototyped in ABS (acrylonitrile butadiene styrene) plastic are presented. The twelve nozzles result from a combination of $15^{\circ}, 30^{\circ}, 45^{\circ}, 60^{\circ}$ radial components and $0^{\circ}, 30^{\circ}, 60^{\circ}$ tangential

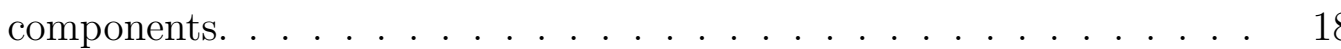

3.1 Velocity pathlines and corresponding shear stress plots are shown for a fixed geometry of $60^{\circ}$ tangental component and $15^{\circ}$ radial component. Shear stress plots are evaluated at the impinged surface. Pathlines are released from the positive pressure inlet and tracked until they exit the computational domain. . . . . . . . . . . . . . . .

3.2 Velocity pathlines and corresponding shear stress plots are shown for a fixed pressure of $10 \mathrm{kPa}$ inlet and $-40 \mathrm{kPa}$ outlet. Shear stress plots are evaluated at the impinged surface. Pathlines are released from the positive pressure inlet and tracked until they exit the domain. . . .

3.3 Velocity pathlines and corresponding shear stress plots are shown for a fixed pressure of $10 \mathrm{kPa}$ inlet and $-40 \mathrm{kPa}$ outlet. Shear stress plots are evaluated at the impinged surface. Pathlines are released from the positive pressure inlet and tracked until they exit the domain. . . .

3.4 A radial distribution of shear stress on the impinged surface is shown for various tangential components and a fixed $15^{\circ}$ radial component. Inlet and outlet pressures are held constant at $10 \mathrm{kPa}$ and $-40 \mathrm{kPa}$ respectively for all combinations. Maximum shear stress is present for a geometry of $60^{\circ}$ tangential component and $15^{\circ}$ radial component. The dashed lines indicate the minimum shear stress required to initiate particle rolling. . . . . . . . . . . . . . . . 
3.5 A radial distribution of shear stress on the impinged surface is shown for various tangential components and a fixed $30^{\circ}$ radial component. Inlet and outlet pressures are held constant at $10 \mathrm{kPa}$ and $-40 \mathrm{kPa}$ respectively for all combinations. The dashed lines indicate the minimum shear stress required to initiate particle rolling. . . . . . . . . . .

3.6 A radial distribution of shear stress on the impinged surface is shown for various tangential components and a fixed $45^{\circ}$ radial component. Inlet and outlet pressures are held constant at $10 \mathrm{kPa}$ and $-40 \mathrm{kPa}$ respectively for all combinations. The dashed lines indicate the minimum shear stress required to initiate particle rolling. . . . . . . . . . .

3.7 A radial distribution of shear stress on the impinged surface is shown for various tangential components and a fixed $60^{\circ}$ radial component. Inlet and outlet pressures are held constant at $10 \mathrm{kPa}$ and $-40 \mathrm{kPa}$ respectively for all combinations. The dashed lines indicate the minimum shear stress required to initiate particle rolling. . . . . . . . . . .

3.8 A radial distribution of shear stress on the impinged surface is shown for a constant geometry of $60^{\circ}$ tangential component and $15^{\circ}$ radial component. The inlet pressure is held at a constant $10 \mathrm{kPa}$ and the outlet pressure ranges form $-30 \mathrm{kPa}$ to $-50 \mathrm{kPa}$ in increments of $10 \mathrm{kPa}$. Maximum shear stress occurs at a condition of $10 \mathrm{kPa}$ inlet pressure and $-40 \mathrm{kPa}$ outlet pressure. The dashed lines indicate the minimum shear stress required to initiate particle rolling. . . . . . . . . . 
3.9 Average and peak shear stress on the impinged surface is shown for a constant geometry of $60^{\circ}$ tangential component and $15^{\circ}$ radial component. The inlet pressure is held at a constant $10 \mathrm{kPa}$ and the outlet pressure ranges form $-10 \mathrm{kPa}$ to $-70 \mathrm{kPa}$ in increments of $10 \mathrm{kPa}$. A maximum average shear stress and maximum peak shear stress occurs at a condition of $10 \mathrm{kPa}$ inlet pressure and $-40 \mathrm{kPa}$ outlet pressure. .

3.10 All parametric permutations are summarized as a function of peak shear stress. The optimal configuration is shown in bold. . . . . . .

3.11 Azimuthal variation is shown for two perpendicular radial shear stress distributions. The distributions correspond the the optimal configuration of $60^{\circ}$ tangential component and $15^{\circ}$ radial component. A slight variation can be seen in the peak shear stress values. The dashed lines represent the outer diameter of the nozzle face. . . . . . . . . . . . 37

3.12 Velocity vectors are shown for a geometry of $60^{\circ}$ tangental component and $15^{\circ}$ radial component. The inlet pressure is defined as $10 \mathrm{kPa}$ and outlet pressure is defined as $-40 \mathrm{kPa}$. The background plot is flooded with contours of velocity magnitude. The velocity vectors depict fluid motion from the positive pressure jets to the impinged surface and out through the negative pressure port. . . . . . . . . . . .

3.13 Velocity vectors are shown at impinged surface for a geometry of $60^{\circ}$ tangental component and $15^{\circ}$ radial component. The inlet pressure is defined as $10 \mathrm{kPa}$ and outlet pressure is defined as $-40 \mathrm{kPa}$. A clockwise rotating vortex pattern can be seen centered about the nozzle's

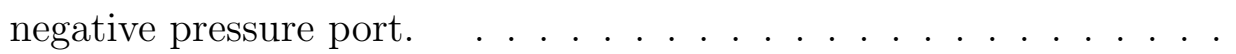


3.14 An iso-surface of vorticity is shown for a geometry of $60^{\circ}$ tangental component and $15^{\circ}$ radial component. The inlet pressure is defined as $10 \mathrm{kPa}$ and outlet pressure is defined as $-40 \mathrm{kPa}$. The iso-surface is drawn at a vorticity magnitude of 15,000. Contour plots of vorticity are depicted on the impinged surface and a vertical plane intersecting the axis of rotation. . . . . . . . . . . . . . .

3.15 Stress lines are shown along the impinged surface for a geometry of $60^{\circ}$ tangental component and $15^{\circ}$ radial component. The inlet pressure is defined as $10 \mathrm{kPa}$ and outlet pressure is defined as $-40 \mathrm{kPa}$. A contour plot of wall shear stress is depicted on the impinged surface. Nearly all enhanced surface shear stress is contained within the annular transition region. The dashed red line represents the circumference of the nozzle

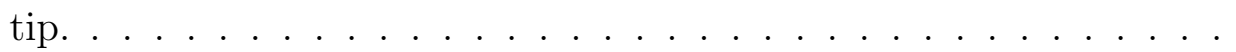

3.16 A plot of surface shear stress is shown for a geometry of $60^{\circ}$ tangental component and $15^{\circ}$ radial component. The applied pressure was fixed at a 1:4 inlet to outlet ratio. The inlet pressure varied from $1 \mathrm{kPa}$ to $10 \mathrm{kPa}$ while the outlet pressure was adjusted to maintain the desired 1:4 ratio. $\ldots \ldots \ldots \ldots . . . \cdots \cdots \cdot \ldots$

3.17 A plot of surface shear stress is shown for varying nozzle heights. The applied inlet pressure is prescribed at $10 \mathrm{kPa}$ and outlet pressure is defined as $-40 \mathrm{kPa}$. A nonlinear relation between nozzle heigh and surface shear stress is shown. . . . . . . . . . . . . .

3.18 Left: The experimental apparatus and nozzle are suspended $10 \mathrm{~mm}$ above an acrylic substrate evenly coated with Lunar simulant (JSC-II). Right: The acrylic substrate and Lunar simulant after only negative pressure is applied to the nozzle's central port. . . . . . . . . . 
3.19 Left: The acrylic substrate and Lunar simulant after only positive pressure is applied to the ring of outer jets. Right: The acrylic substrate and Lunar simulant after positive and negative pressure is simultaneously applied to the ring of outer jets and central port respectively. .

3.20 A progressive set of photographs depicting the particle removal process is shown. The impingement surface is imaged from the underside of a transparent impingement surface. Initially the surface is evenly covered with a regolith simulant. As negative and positive pressures are applied, the particles are dislodged and removed from the impingement surface. As particles are removed, the surface of the nozzle and ports become visible. . . . . . . . . . . . . . . . .

3.21 The experimental high-speed photography apparatus consists of a prototype nozzle positioned above a glass impingement surface. The removal process is imaged from beneath the impingement surface using a high-speed camera. The left image shows the surface before particle removal. The right image illustrates the surface after the removal

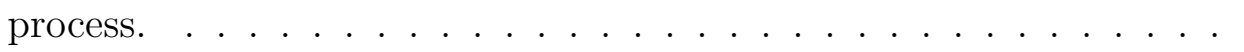

4.1 Critical shear stress is shown as a function of particle size. Particle size ranges from 10 micron to 100 micron. Critical shear stress scales nonlinearly with particle size. . . . . . . . . . . .

4.2 A particle size distribution is shown for Lunar simulant JSC-1A. The distribution was calculated by NASA using a laser diffraction particle size analyzer $[19] . \ldots \ldots \ldots$. . . . . . . . . . . . . 54

4.3 A radial shear stress distribution for the optimal nozzle configuration and pressure condition is shown with an overlay of critical shear stress. 
4.4 A comparison between the experimental removal radius and the predicted radius is shown. The experimental radius is shown in red and the predicted radius is shown in blue. . . . . . . . . . . . .

A.1 A technical drawing of the experimental nozzle assembly is shown. The lower portion of the nozzle can be detached and exchanged with an alternative tangential and radial configuration. A $0^{\circ}$ tangential component and $15^{\circ}$ radial component is depicted. . . . . . . . . . . . 70

A.2 A technical drawing of the 'optimal' experimental nozzle is shown. A $60^{\circ}$ tangential component and $15^{\circ}$ radial component is depicted. . . . 


\section{Chapter 1}

\section{Introduction}

Methods of dust mitigation in Martian and Lunar environments are becoming an increasingly important consideration in light of future space missions to the Moon and Mars. Unlike Earth, the Moon and Mars lack aqueous precipitation and egress, which would aid in the removal of dust aggregate on exposed surfaces. The absence of moisture in Martian and Lunar environments results in a significant buildup of electrical charge on particles. In Martian environments the charge is acquired through triboelectric charging [14]. In Lunar environments solar radiation is the main charging mechanism [1]. The significant static charge causes particles to strongly adhere to surfaces, such as solar panels, space suits, clothing, and mechanical devices [18].

Particles remain sharp and abrasive due to an absence of erosion characteristics present in Earth's environment. An image of Lunar simulant particles under an optical microscope can be seen in Figure 1.1. The abrasive characteristics of particles can greatly reduce the projected life of equipment through frictional forces. In addition to mechanical failure, particles may pose a serious health risk. During the Apollo 17 mission to the Moon, electrically charged lunar regolith had adhered to spacesuits during extra-vehicular activities on the Moon's surface. Upon reentering the Lunar 
Module, particles became airborne and were inhaled by the occupying astronauts. Symptoms of congestion, fever and a "Gun Powder" smell were reported [20] . In future Martian and Lunar missions, dust mitigation will be paramount in preserving human health and mechanical longevity [10].

\subsection{Existing Mitigation Strategies}

Charged and uncharged particles can be removed from surfaces using electrostatic forces. Calle et al. (2008) have had success in transporting particles using an arrangement of parallel conductors [5]. An AC source is applied to the conductors to generate a traveling electrostatic wave. The wave acts as a conveyer causing particles to travel along or against the direction of the wave. The system is effective at clearing a specified area but further particle removal may be required to completely remove particles from the contaminated object. Variations of this method have been applied to clean surfaces such as solar panels.

Acoustic radiation in conjunction with air impingement has been used by Chen and $\mathrm{Wu}(2009)$ to dislodge particles from various surfaces [6]. Acoustic radiation at a frequency of $13.8 \mathrm{kHz}$ and pressure of $128 \mathrm{~dB}$ was delivered to a surface using a $3 \mathrm{~cm}$ aperture tweeter. A standing wave was maintained between the tweeter and reflector surface. It was discovered that this radiation is sufficiently strong to overcome van der Waals adhesive force between dust particles and the surface. Once particles were levitated, an air flow was applied to remove the particles from the surface.

Lu et al. (1999) studied the effects of Laser Pulses on the removal of micron sized particles [15]. The method has been utilized to remove particles from optical apparatus, archive portraits, and metal surfaces. During the laser pulse, the particle gains acceleration through sudden surface and particle expansion. The acceleration 
can be millions of times larger than earth's gravitational acceleration. Particles break free of adhesive bonds and are dislodged from the surface. Particle removal efficiency increase with an increase in laser intensity. It was concluded that the laser intensity threshold required to remove particle increases abruptly for particles less than 1 micron.

As summarized by Hyatt et al. (2007), several conventional methods of dust mitigation were used in the Apollo surface operations [10]. A water jet and wet cloths were used to clean suits, boots, and gloves. A lens brush was used to clean camera optics. A vacuum cleaner was used to remove dust from the living environment. Stomping, brushing the suit, and placing bags over the boots upon reentry reduced dust infiltration. Repeated wiping of surfaces easily scratched visors and displays rendering them dysfunctional.

\subsection{Surface Shear Stress}

Particle removal strongly depends on the magnitude of shear stress imposed on the target surface. Enhanced surface shear stress can be achieved using a variety of methods. Jet impingement is one common strategy used to impose shear stress and remove particles from surfaces. Also, many methods associated with surface cooling can be applied to enhance surface shear stress [29].

Zhang et al. (2002) experimentally characterized particle removal efficiency of a single air jet delivered by a $3 \mathrm{~mm}$ converging nozzle. Glass spheres (40-50 micron) were applied to the test surface and removal efficiency was calculated after jet impingement. It was determined that removal efficiency strongly depends on the distance from the surface, pressure applied, elapsed time, and impingement angle. For

a single jet, a 30 degree impingement angle resulted in the greatest particle removal. 
The minimum pressure required to provide particle transport depended on surface roughness and particle properties. A critical pressure exists beyond which little particle removal efficiency is gained. Maximum removal efficiencies were reached for impinging distances of $40 \mathrm{~mm}$ (13 diameters) to $60 \mathrm{~mm}$ (20 diameters) [28].

Masuda et al. (1994) and Ziskind et al. (2002) studied particle removal from surfaces by pulsed air jet impingement [17], [29]. Pulsed flow was produced using an electromagnetic valve in Masuda's work and a chopper wheel in Ziskind's experiment. It was determined that a critical range of frequencies are most effective at dislodging particles from surfaces. An optimal impingement angle of 30 degrees was identified for producing the greatest particle removal. This result agrees with the continuous air jet study performed by Zhang et al. (2002).

Shock tube vortex ring impingement as studied by Kontis et al. (2008) can be used to induce surface shear stress [13]. A vortex ring was generated using an open ended shock tube with a $30 \mathrm{~mm}$ internal diameter. As the vortex ring approaches the wall the flow accelerates rapidly in the radial direction imposing shear stress on the impinged surface. Such flow could be effective in dislodging strongly adhered particles.

Parras et al. (2007) studied the interaction of an unconfined vortex with a solid surface [21]. The interaction of a vortex with a solid plane perpendicular to its axis of rotation was solved numerically. It was determined that the swirl intensifies as the vortex approaches the impinged surface. The flow resembled intense tornado-like vortices where a significant annular updraft was present. The flow at the axis of the vortex was directed downward. This intensified flow could enhance shear stress on the surface. 


\subsection{Enhanced Flow Structures}

The interaction of a vortex with a ridged wall was studied by Hirsa et al. (2000) [9]. A vortex was generated via a pair of rotating flaps in a cylindrical water tank. The flow structure was observed using a combination of planar laser-induced florescence and digital particle image velocimetry. It was found that a toroidal recirculation region forms when a vortex interacts with a no-slip wall. The toroidal structure was not the result of the vortex itself but instead the result of the end wall boundary structure.

Phillips et al. (1987) experimentally investigated turbulent vortex boundary layers [22]. A vortex was generated by rotating a cylindrical water tank about its axis of symmetry. A stationary surface was positioned in the bottom of the tank to provide a no-slip boundary. Through the use of laser-Doppler anemometer, radial meanvelocity distributions were captured. Reynolds number flows ranging from 5,000 to 30,000 were explored. Two opposing factors were determined to effect the stability of the vortical flow. The inflection point in the crossflow velocity profile acts as a source of dynamic instability, while the pressure gradient acts to stabilize the flow. It was concluded that at high enough $R e$ the pressure gradient near the surface will not be able to overcome the dynamic instability. As a result the flow will become and remain turbulent.

Wen et al. (2003) studied the impingement of a swirling jet on a flat surface [27]. A tube with a helical insert provided a means of producing swirling flow. The tube was positioned above a heated impingement surface. A vortical flow was produced on the impingement surface by the swirling flow developed in the tube. The cooling effects were evaluated both with and without a swirling insert. Reynolds numbers between 500 and 27,000 were investigated. Smoke flow visualization was implemented to visualize the flow behavior of the impinging jet. The swirling flow was found to provide 
superior surface cooling performance. It was observed that the cooling performance increased with and increase in Reynolds number. Heat transfer enhancement varied between $9 \%$ and $14 \%$.

Swirling jet impingement on a flat plate was characterized by Abrantes et al. (2006) [2]. A swirling flow was produced using an array of tangentially angled ports in a swirl generating cylinder. The flow was then passed through a $22 \mathrm{~mm}$ tube where it was directed at a flat plate. Swirl intensity was adjusted by making the tube longer. Jet to plate distances of 2 and 0.25 times the jet diameter were explored. All experimental configurations correspond to a Reynolds number of 21,000. The swirl velocity on the impinged surface significantly impacts the local heat transfer distribution. For a $44 \mathrm{~mm}$ jet-to-plate spacing, the added swirl caused fluctuations in the heat transfer distribution and a large recirculating flow region at the jet stagnation region. The result was a decrease in heat transfer. For a $5.5 \mathrm{~mm}$ jet-to-plate spacing, the presence of swirl produced an enhancement of heat transfer from the plate. The enhanced heat transfer was contributed to the stronger axial momentum that precluded the formation of the recirculating flow zone at the stagnation region and the stronger circumferential component.

The impingement of a swirling jet on a heated flat plate has also been studied by Ichimiya et al. (2010) at lower Reynolds numbers [12]. Flow and heat transfer characteristics were gathered both numerically and experimentally. Temperature distribution and velocity vectors were gathered at various locations from the nozzle exit to the impinged surface. Numerous swirl numbers were evaluated for flows with a Reynolds number of 2,000. A swirling impinging jet was determined to both enhance and depress heat transfer. Flow acceleration and flow mixing near the impinged surface caused enhanced heat transfer. Rising and recirculating flow depressed heat transfer. Heat transfer on the impinged surface reached a maximum at a specific swirl 
number.

\subsection{Bound Vortex Flow}

In this study we investigate an active mechanism designed to enhance the removal of particles adhered to surfaces. A nozzle configuration was designed to generate a bound vortex flow condition. Through the impingement of a bound vortex, shear stress on a surface is amplified, increasing particle transportation. Dislodged particles can be drawn through a central negative pressure port and safely contained. Using computational fluid dynamic simulations, the effectiveness of various vortex-inducing conditions is evaluated. Shear stress on the impinged surface is used to indirectly evaluate particle removal capacity. The effect of particle charge on the removal process is neglected in this study. Through a parametric study, an optimal range of key geometric and pressure parameters are identified to create bound vortex flow. A set of computational conditions were recreated experimentally to confirm vortex formation and enhanced particle removal. 


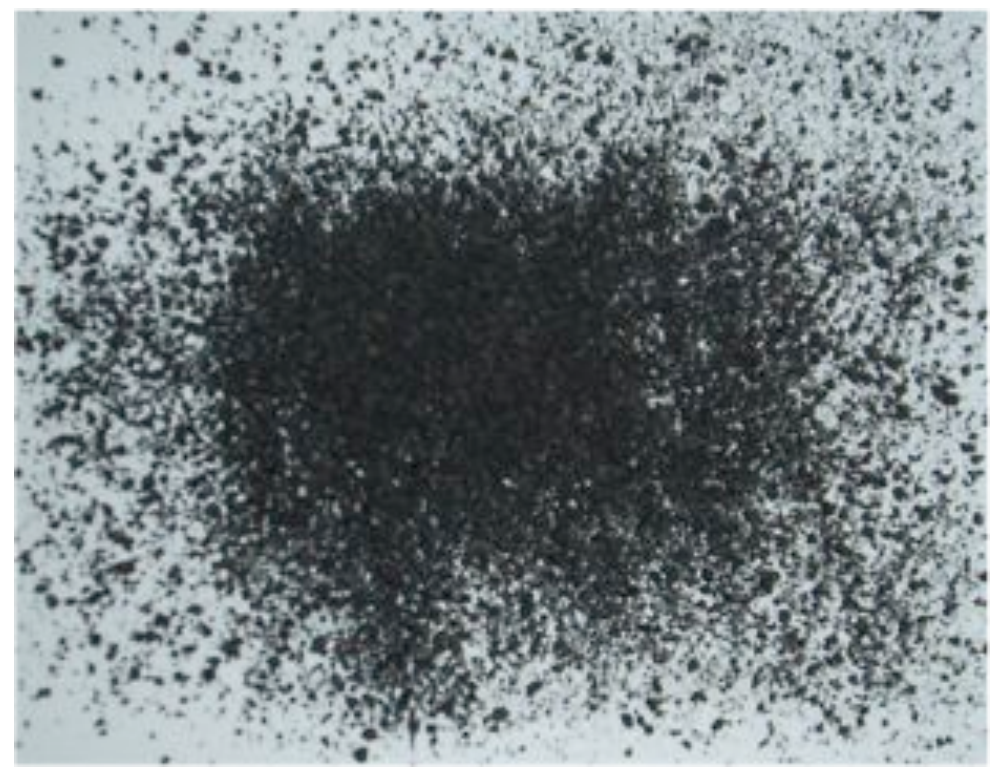

(a) A photograph of lunar regolith simulant (JSC-II)

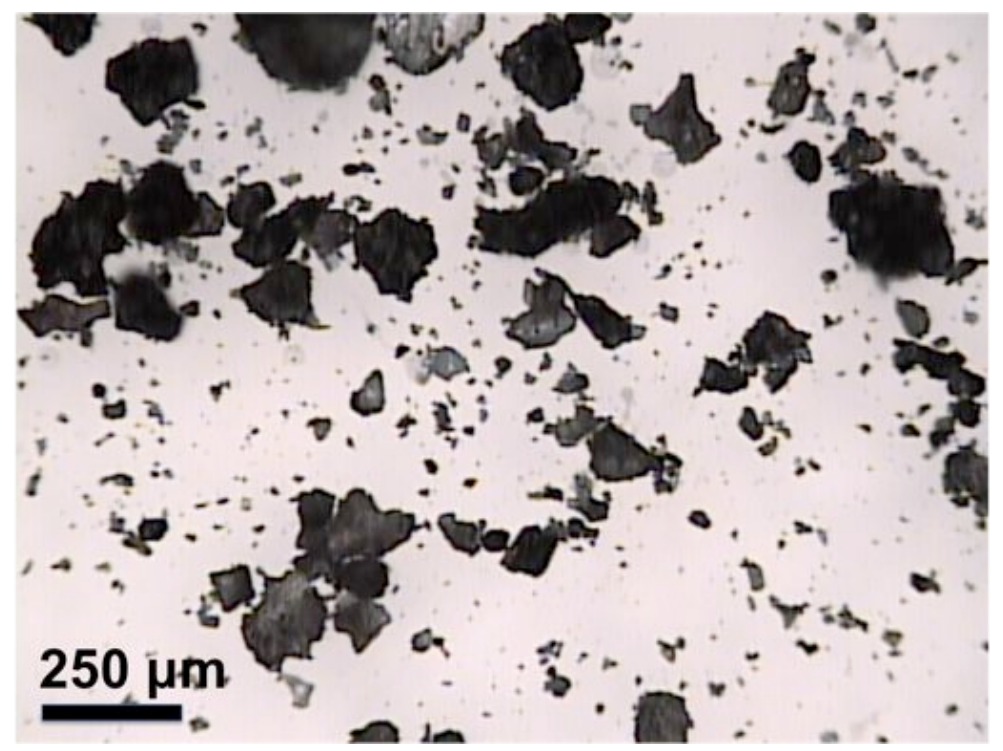

(b) An image of lunar regolith simulant (JSC-II) under an optical microscope

Figure 1.1: Lunar regolith simulant particles are nonuniform, sharp, and abrasive. Martian and Lunar dust particles can negatively impact human health and reduce the mechanical longevity of equipment. 


\section{Chapter 2}

\section{Methodology}

\subsection{Nozzle Design}

The essential element in the bound vortex generation is a complex nozzle featuring a combination of positive pressure inlets and a central negative pressure outlet (Figure 2.1). Acting together, this combination of positive and negative pressure inlets/outlet establishes, with proper parametric conditions, the possibility of a closed flow structure (bound vortex). For the inlet ports, both the radial and tangential components represent independent geometric features of the nozzle that can be used to control and refine the structure of the vortical flow. To see this, consider the radial component of the inlet interacting with the negative pressure core: the radial angle will clearly impact the extent of the vortex beneath the nozzle. The role of the tangential component is, in contrast, to add swirl to the flow structure. In this work it is posited that there exists an optimal configuration(s) that maximize shear stress on a surface interacting with the bound vortex.

To explore the nozzle operating characteristics and performance, computational domains were developed for a broad range of bound vortex flow conditions. As seen 
in Figure 2.1, each nozzle consists of a central negative pressure port surrounded by an array of positive pressure jets. A total of twelve nozzles were created, each share the common characteristic of a single $5 \mathrm{~mm}$ central port and ten equally spaced 1.5 $\mathrm{mm}$ jets which are aligned along a circle $15 \mathrm{~mm}$ in diameter. The height of nozzle above the impingement surface was chosen to be $10 \mathrm{~mm}$, as it would provide adequate surface flow interaction. Variations in nozzle geometry were determined by the radial and tangential components of the jet array. Radial components of $15^{\circ}, 30^{\circ}, 45^{\circ}, 60^{\circ}$ and tangential components of $0^{\circ}, 30^{\circ}, 60^{\circ}$ were explored.

Initial nozzle geometries were created in SolidWorks Corp.'s SolidWorks 2009 three-dimensional solid modeling software. SolidWorks was chosen for its ability to create and quickly modify complex models parametrically. Nozzle geometries were exported as a STEP file and imported into ANSYS Inc.'s GAMBIT 2.1 grid generation software.

A computational domain was created around each nozzle geometry. The domain consisted of a cylinder $80 \mathrm{~mm}$ in diameter, centered on the nozzle and extending $10 \mathrm{~mm}$ beyond the nozzle tip. The size of the domain was chosen to insure all surface impingement characteristics were captured. The size of the domain was chosen to ensure the absence of boundary effects in the flow field region of interest. A Boolean subtraction was applied to remove the positive nozzle geometry from the computational domain resulting in a purely fluid domain. The computational domain was sub-divided to facilitate flexibility in the grid refinement process. Tetrahedral elements were applied throughout the entire domain. The number of tetrahedral elements ranged from approximately 3,500,000 to 4,000,000 due to variations in nozzle geometry. An example of a completed grid can be seen in Figure 2.2, which depicts varying tetrahedral grid spacing with the greatest refinement at the nozzle exit. 


\subsection{Computational Model}

The completed grid was imported into ANSYS Inc.'s Fluent 6.3 computational fluid dynamics flow modeling software to obtain a solution to the governing incompressible Navier-Stokes equations (Appendix A.1). Reynolds numbers were calculated for the proposed range of inlet and outlet pressures and determined to reside within the turbulent regime. Small density changes and relativity low velocities motivated the choice of an incompressible model.

A realizable $k-\epsilon$ turbulence model was utilized for its superior performance in flows involving rotation, vortices, recirculation, and strong streamline curvature. The standard $k-\epsilon$ model, developed by Launder and Spalding (1972), is a semi-empirical model based on model transport equations for the turbulence kinetic energy $(k)$ and its dissipation rate $(\epsilon)$ [16]. The model, developed by Shih et al. (1995), differs from the standard $k-\epsilon$ model in that it contains a new formulation for the dissipation rate equation and a new realizable eddy viscosity formulation [25]. The dissipation equation is based on the dynamic equation of the mean-square vorticity fluctuation at large turbulent Reynolds number. The eddy viscosity formulation incorporates the constraint of a positive normal Reynolds stress and the Cauchy-Schwarz inequality for turbulent shear stress. The model improves the numerical stability in turbulent flow calculations when used with a second order closure scheme. It also removes the spreading rate abnormality of planar and round jets, such as the jets present in the bound vortex nozzle design. Further details on the formulation of the realizable $k-\epsilon$ turbulence model can be viewed in Appendix A.2.

The SIMPLE algorithm was used for pressure-velocity coupling and a second-order

upwind scheme was used for spatial discretization. A second-order upwind scheme provided higher-order accuracy at cell faces through a Taylor series expansion of the 
cell-centered solution [3]. Additional details on the Fluent's implementation of the SIMPLE algorithm and second-order upwind scheme can be found in Appendix A.5 and Appendix A.6. A steady state solution was obtained using a pseudo time stepping iterative process. Global residual monitors of Continuity, X-Velocity, Y-Velocity, ZVelocity, Turbulent Kinetic Energy, and Turbulent Dissipation were monitored for convergence. Surface shear stress point monitors were applied along the impinged surface and monitored for convergence.

A pressure inlet and pressure outlet boundary condition was applied to the nozzle jet inlet and central port respectively. Pressure boundary conditions were chosen to accurately represent the final application in which a constant positive or negative pressure would be supplied to the nozzle. Pressures were applied in terms of gauge pressure in contrast to absolute pressure. The upper surface and sides of the cylindrical domain were defined as a pressure outlet of zero gauge pressure to represent a nozzle in an open environment. Internal and external nozzle surfaces and lower impingement surface were defined as walls with a no slip condition. The working fluid for this application was air at a pressure of $101.3 \mathrm{kPa}$ which is intended for our current laboratory conditions; this would also be appropriate for operation within a human occupied Lunar or Martian habitat. Boundary conditions and locations can be viewed in Figure 2.2.

The flow field was initialized to zero velocity for the first computational permutation. The previous converged solution was then used as the initial condition for each successive study. This method decreased computational demand by reducing the iterations required to reach a converged solution.

A formal grid refinement study was performed to ensure that spatial discretization errors were minimized. Average and peak shear stress on the impinged surface was monitored for a constant geometry of $60^{\circ}$ tangential component and $15^{\circ}$ radial com- 
ponent. The inlet pressure was held at $10 \mathrm{kPa}$ and the outlet pressure at $-40 \mathrm{kPa}$. By monitoring average and peak surface shear stress over a range of grid resolutions, it was determined that a resolution of 4,000 elements per meter was sufficient to provide grid independent results. A plot depicting the grid refinement study can be viewed in Figure 2.3.

\subsection{Experimental Methods}

In order to support the numerical simulations, an experimental test fixture was developed to accommodate all nozzle geometries and distribute positive and negative pressures to the nozzle inlet and outlet respectively. A solid model of each nozzle and the experimental fixture were exported from SolidWorks 2009 as a STL (Stereo Lithography) file and prototyped in ABS (Acrylonitrile Butadiene Styrene) plastic using a fused deposition rapid prototype machine (Figure 2.4). Internal nozzle dimensions were chosen to compliment the resolution limitations of the rapid prototype machine. A nozzle with a $30^{\circ}$ tangental and $15^{\circ}$ radial component geometry was attached to the experimental fixture. As seen in Figure 3.18, the fixture and nozzle were suspended $10 \mathrm{~mm}$ over a clear acrylic surface to reproduce the geometry of the computational domain. A Lunar simulant (JSC-II) was evenly applied to an acrylic surface until complete coverage was obtained.

A constant positive and negative pressure source was attached to the test fixture. A set of ball valves allowed for simple on or off control of the pressure sources. A regulator was used to adjust the magnitude of positive pressure. The negative pressure was controlled by diverting a portion of the pressure to the ambient environment through a needle valve. In the first experiment, negative pressure alone was applied to the nozzle. A second experiment consisted solely of positive pressure application. 
The third experiment incorporated a combination of both positive and negative pressure application simultaneously. In each circumstance, pressure was applied until no further particle movement was observed. 

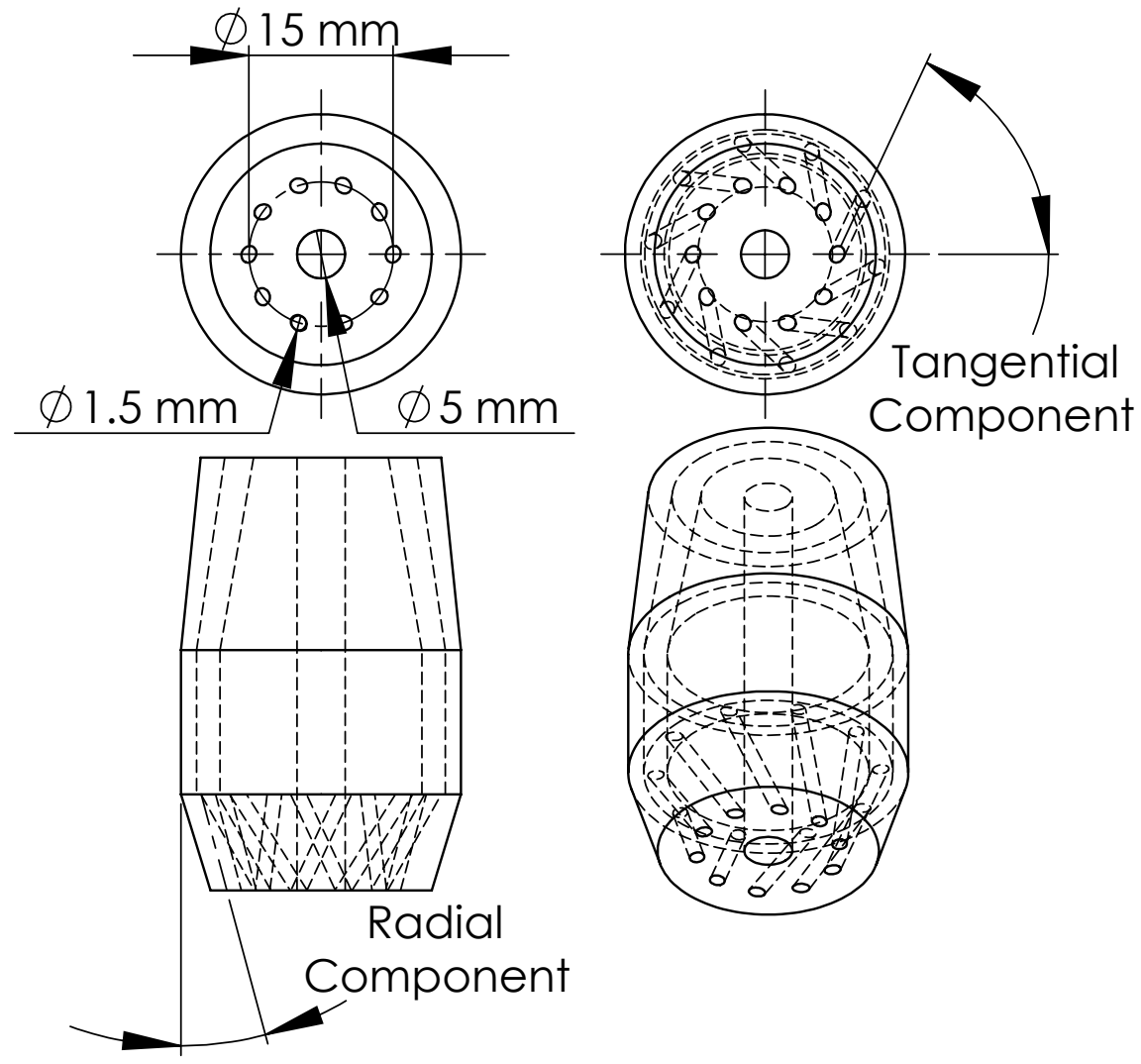

Figure 2.1: A drawing depicting a basic nozzle geometry consisting of a single 5 $\mathrm{mm}$ central port and ten equally spaced $1.5 \mathrm{~mm}$ jets. The positive pressure jets are defined by a tangential and radial component. 


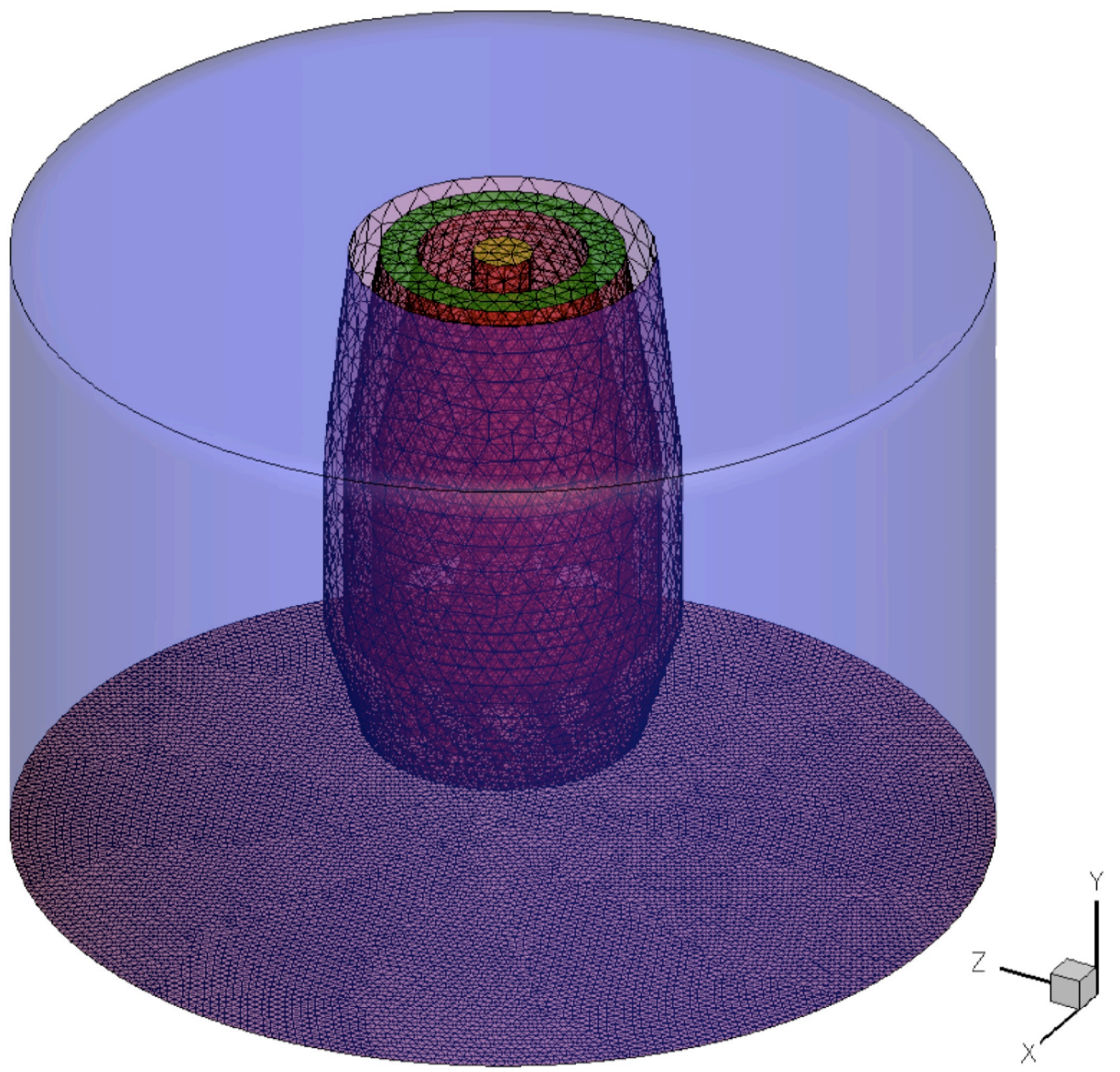

Figure 2.2: A computational grid comprised of tetrahedral elements is shown. Tetrahedral grid density increases within the domain as the impinged surface is approached. Boundary conditions are defined by the following color key: Blue - Pressure Outlet; Red - No-Slip Wall; Green - Positive Pressure Inlet; Yellow - Negative Pressure Outlet. 


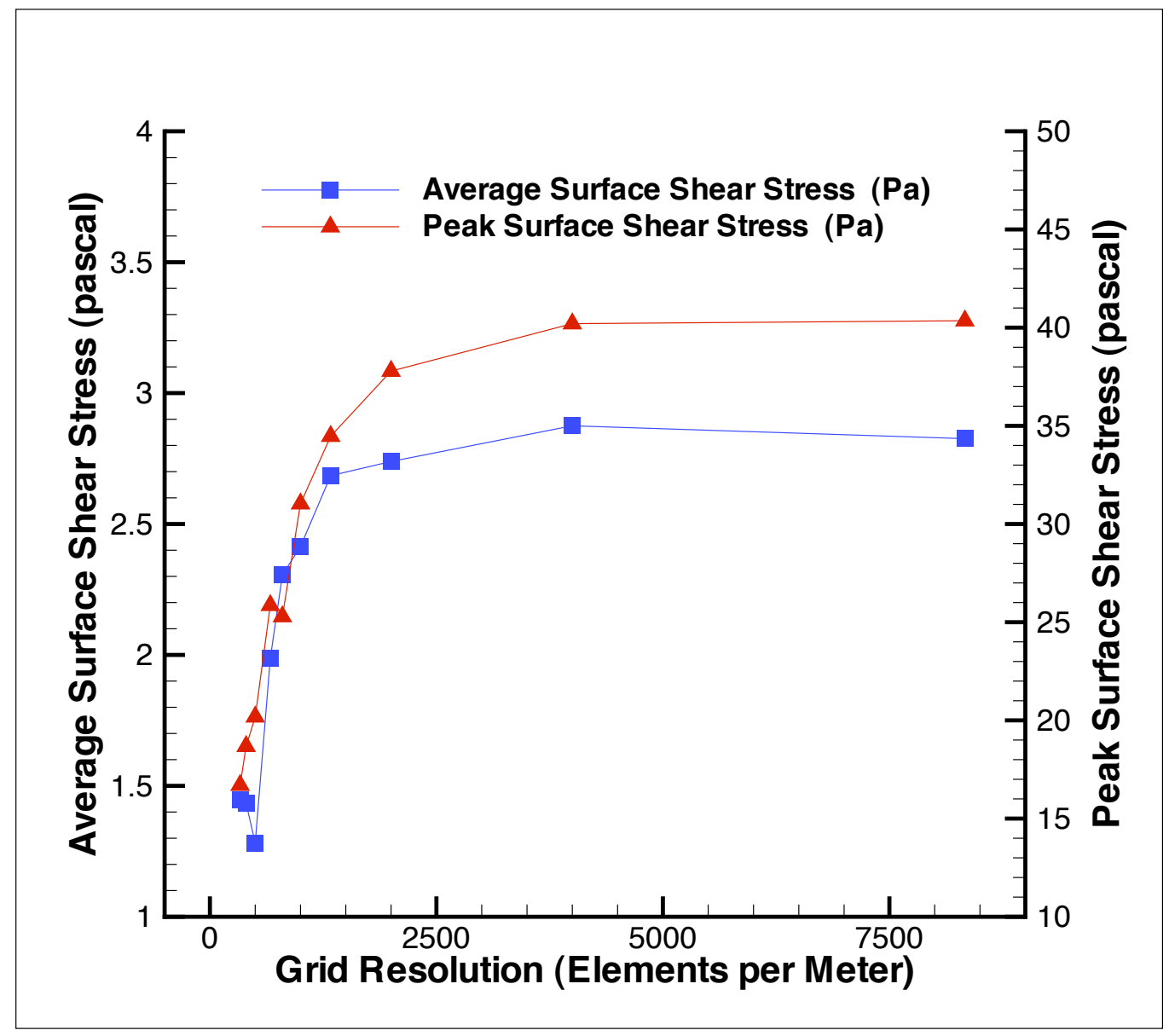

Figure 2.3: Average and peak shear stress on the impinged surface is shown for a constant geometry of $60^{\circ}$ tangential component and $15^{\circ}$ radial component. The inlet pressure is held at a constant $10 \mathrm{kPa}$ and the outlet pressure at a constant $-40 \mathrm{kPa}$. The value of peak and average shear stress remain constant at a grid resolution at or greater than 4000 elements per meter. 


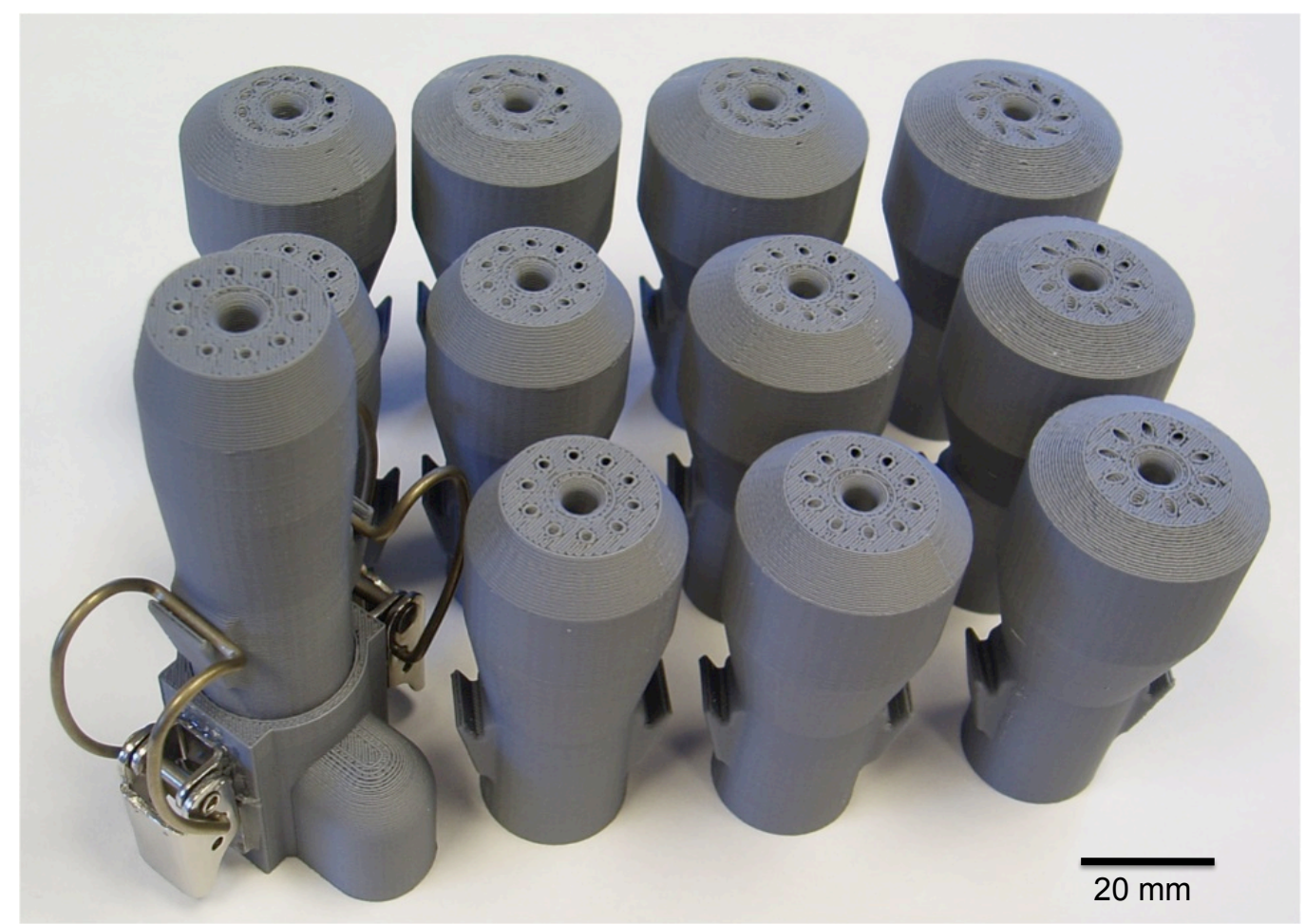

Figure 2.4: Twelve nozzles rapid-prototyped in ABS (acrylonitrile butadiene styrene) plastic are presented. The twelve nozzles result from a combination of $15^{\circ}$, $30^{\circ}, 45^{\circ}, 60^{\circ}$ radial components and $0^{\circ}, 30^{\circ}, 60^{\circ}$ tangential components. 


\section{Chapter 3}

\section{Results}

\subsection{Computational Results}

A parametric study was performed to evaluate the flow characteristics for each nozzle geometry over a range of inlet and outlet gauge pressure conditions. The inlet pressure was held at $10 \mathrm{kPa}$ while the outlet pressure varied from $-10 \mathrm{kPa}$ to $-70 \mathrm{kPa}$ in increments of $10 \mathrm{kPa}$. All nozzle configurations and pressure conditions can be seen in Table 3.1 and Table 3.2 respectively. The nozzle height was fixed at $10 \mathrm{~mm}$ above the surface. The pressure outlet boundary condition on the side and top of the cylindrical domain was set to $0 \mathrm{kPa}$ gauge pressure to simulate a nozzle releasing into an open environment. The lower boundary was prescribed as a no slip wall condition to represent the impinged surface. A converged solution was obtained for each pressure permutation.

Shown in Figure 3.1 are pathline visualizations for the different flow conditions for a fixed nozzle geometry. Also appearing below each pathline visualization is the associated shear stress distribution on the surface. For this case of varying pressure conditions, a fixed geometry of $60^{\circ}$ tangental component and $15^{\circ}$ radial component 
was used.

If a weak negative outlet pressure $(0 \mathrm{kPa}$ to $-20 \mathrm{kPa})$ is applied, flow exits the ring of jets, strikes the surface, and the majority of the flow exits through the side of the domain. This would not be advantageous, as it would cause particles on the impinged surface to accelerate away from the central vacuum port preventing them from being captured. As a result of strong negative outlet pressure $(-50 \mathrm{kPa}$ to $-70 \mathrm{kPa})$, the flow exits the ring of jets and is captured by the central vacuum port before it contacts the lower surface. This is not a beneficial result because it prevents the jets from inducing higher shear stress at the impinged surface, limiting particle transportation. If a medium negative outlet pressure $(-20 \mathrm{kPa}$ to $-50 \mathrm{kPa})$ is applied, the flow exits the ring of jets, creates a bounded vortex, impinges on the surface and exits the central port. Bound vortex impingement increases surface shear stress, which enhances the removal of particles adhered to the surface. The vortex would dislodge, levitate and entrain loosened particles while the central port applies the negative pressure required to remove them.

Next, the corresponding pathline and surface shear stress distributions have been computed for different nozzle geometries with a fixed pressure condition of $10 \mathrm{kPa}$ inlet and $-40 \mathrm{kPa}$ outlet (Figures 3.2 and 3.3). Bound vortex formation has a clear dependence on radial and tangential jet components. In permutations with zero tangential components, no vortex was formed. The rotational strength of the vortex increased with an increase in the tangential jet component. The radial component dictated the depth of vortex penetration. As the radial component increased, the vortex deviated less from the nozzle exit plane.

For the optimal geometry suggested from Figures 3.4, 3.5, 3.6, and 3.7, shear stress distributions were then computed for several outlet conditions and appear in Figure 3.8. This data has been further reduced to key statistics of mean and peak shear stress 
in Figure 3.9. Shear stress created at the impinged surface provides the mechanism for enhanced particle removal [29]. From this data it is found that an overall optimizing condition exists for this nozzle height of $10 \mathrm{~mm}$. A nozzle with a $60^{\circ}$ tangental and $15^{\circ}$ radial component provided the greatest shear stress while maintaining a controllable axisymmetric vortex. Average and peak stress distributions presented in Figure 3.8 confirm that both average and peak shear stress over the impinged surface reach a maximum at the optimum pressure condition of $10 \mathrm{kPa}$ inlet and $-40 \mathrm{kPa}$ outlet.

A depiction of all 84 permutations is summarized as a function of peak shear stress in Figure 3.10. The optimal nozzle configuration far outperforms the remaining 11 designs. A plateau is formed between the outlet pressures of $-40 \mathrm{kPa}$ and $-60 \mathrm{kPa}$ with a maximum peak shear stress at a condition of $-40 \mathrm{kPa}$.

The bi-peaked radial shear stress distribution is largely axisymmetric but does contain a slight azimuthal variation. Figure 3.11 shows two radial shear stress distributions along the impinged surface for the optimal configuration. One distribution is along a 0 degree azimuthal direction and the other is along a 90 degree direction. A variation in the peak shear stress value is most apparent. While little variation is seen in the remainder of the shear stress distribution.

Additional flow visualization for the optimal geometric and pressure configuration is provided by Figure 3.12 and Figure 3.13. Velocity vectors perpendicular to the impingement surface and parallel to the nozzle's axis of symmetry clearly depict fluid motion from the positive pressure jets to the impinged surface and out through the negative pressure port. Toroidal flow patterns can be seen each side of the nozzle's axis of symmetry in Figure 3.12. Velocity vectors at the impinged surface represent a classic vortex pattern centered about the nozzle's axis (Figure 3.13).

For the optimal configuration and pressure condition, an iso-surface plot of vor- 
ticity is shown in Figure 3.14. A well defined central vortex can be seen between the nozzle exit and impinged surface. Contours plots of vorticity are also depicted on the impinged surface and a vertical plane intersecting the axis of rotation. A nearly axisymmetric vorticity pattern can be seen on the impinged surface with the greatest magnitude present at the vortex core.

An annular transition region exists on the impinged surface, at which the shear stress acting on the surface changes from an inward radial component to an outward radial component. This region can be seen in Figure 3.15, which depicts stress lines on the impinged surface for the optimal nozzle configuration and pressure condition. The transition region is nearly axisymmetric and resides just outside the face of the nozzle. The region coincides with a toroidal vortex formation shown in Figure 3.12. The wall shear stress contour plot, seen in Figure 3.15, is presented on the impinged surface where the circumference of the nozzle's exit face is shown as a dashed red line. The vast majority of enhanced shear stress resides interior to the transition region. Consequently, particle removal and transportation is limited outside the transition region and the dominate removal mechanism is in the inward radial direction.

Having determined an optimal geometric and pressure condition, a study was performed to characterize the effect of pressure magnitude. A fixed pressure ratio of 1 inlet to 4 outlet was chosen and applied to a geometry of $15^{\circ}$ radial component and $60^{\circ}$ tangential component. The assigned inlet pressure varied from $1 \mathrm{kPa}$ to $10 \mathrm{kPa}$ in increments of $1 \mathrm{kPa}$. The outlet pressure varied from $4 \mathrm{kPa}$ to $40 \mathrm{kPa}$ with increments chosen to maintain the desired 1:4 ratio. Pressure conditions and corresponding Reynolds numbers are shown in Table 3.3. Reynolds numbers were calculated for the positive pressure jets based on a diameter of $1.5 \mathrm{~mm}$. A plot depicting shear stress as a function of pressure magnitude can be seen in Figure 3.16. The relationship between the magnitude of pressure applied and shear stress on the 
impinged surface was found to be nearly linear.

A study was also performed to evaluate the effect of nozzle impingement height on surface shear stress. Figure 3.17 depicts both peak and average surface shear stress as a function of nozzle-to-surface height, where an inlet pressure of $10 \mathrm{kPa}$ and outlet pressure of $-40 \mathrm{kPa}$ were applied. Nozzle-to-surface height ranged from $2 \mathrm{~mm}$ to 20 $\mathrm{mm}$ in increments of $2 \mathrm{~mm}$. A nonlinear relationship between height and shear stress can be seen for both average and peak shear stresses. Surface shear stress increased rapidly as nozzle height decreased.

\subsection{Experimental Results}

Experimental results indicate that the addition of bound vortex flow clearly has a positive effect on the removal of particles from a surface. Lunar simulant particles remained adhered to the acrylic substrate when only negative pressure was applied to the nozzle's central port, as seen in Figure 3.18. Almost no particle movement was observed, apart from slight particle oscillations near the central port. When only positive pressure was applied to the ring of outer jets, particles were violently accelerated away from the center of the nozzle. Particles were thrown far from the impinged surface, as seen in Figure 3.19. When positive pressure was applied to the ring of outer jets in conjunction with negative pressure applied to the central port, particles were dislodged and removed through the central port. In approximately 1 to 2 seconds, particles were removed from the acrylic surface leaving a clear circular pattern, as seen in Figure 3.19.

The flow velocities and particle removal process occur much too rapidly to capture via standard video frame rates. The use of a high-speed camera is needed to provide sufficient resolution to capture particle lift-off and entrainment. Through the use of 
high-speed photography, the evolution of particle removal was captured. Frame rates ranging from 60 to 2000 frames per second were explored. Lower frame rates were well suited to visualizing the progression of particle removal as seen in Figure 3.20, which depicts the scouring of a layer of dust from a transparent surface. Higher frame rates allowed individual particles to be tracked from the impingement surface to the nozzle outlet. The high-speed experimental apparatus is shown in Figure 3.21. 
Table 3.1: Geometric nozzle parameters resulting in 12 nozzle configurations.

\begin{tabular}{|c||c|c|}
\hline \multicolumn{2}{|c|}{ Nozzle Configurations } \\
\hline Configuration & Tangential Component $\left(^{\circ}\right)$ & Radial Component $\left(^{\circ}\right)$ \\
\hline 1 & 0 & 15 \\
2 & 0 & 30 \\
3 & 0 & 45 \\
4 & 0 & 60 \\
5 & 30 & 15 \\
6 & 30 & 30 \\
7 & 30 & 45 \\
8 & 60 & 60 \\
9 & 60 & 15 \\
10 & 60 & 30 \\
11 & 60 & 45 \\
\hline
\end{tabular}


Table 3.2: Seven pressure conditions applied to each nozzle.

\begin{tabular}{|c||c|c|}
\hline \multicolumn{3}{|c|}{ Pressure Conditions } \\
\hline Pressure Condition & Inlet Pressure $(\mathrm{kPa})$ & Outlet Pressure $(\mathrm{kPa})$ \\
\hline 1 & 10 & -10 \\
2 & 10 & -20 \\
3 & 10 & -30 \\
4 & 10 & -40 \\
5 & 10 & -50 \\
6 & 10 & -60 \\
7 & 10 & -70 \\
\hline
\end{tabular}



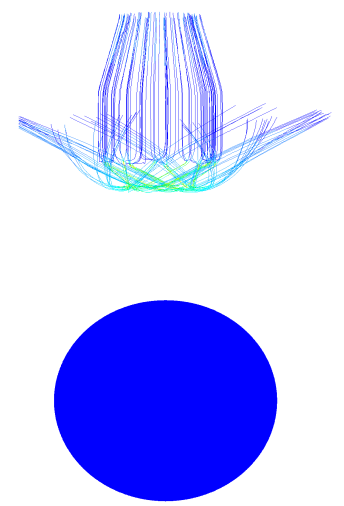

(a) $10 \mathrm{kPa}$ inlet, $-10 \mathrm{kPa}$ outlet
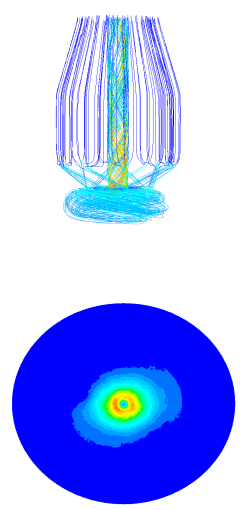

(d) $10 \mathrm{kPa}$ inlet, $-40 \mathrm{kPa}$ outlet
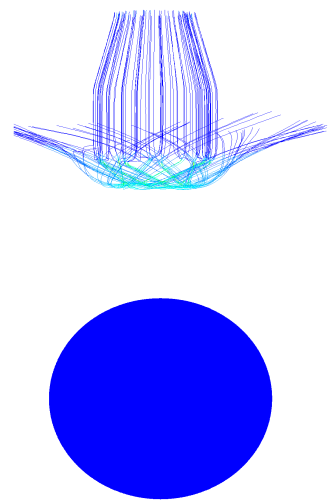

(b) $10 \mathrm{kPa}$ inlet, $-20 \mathrm{kPa}$ outlet
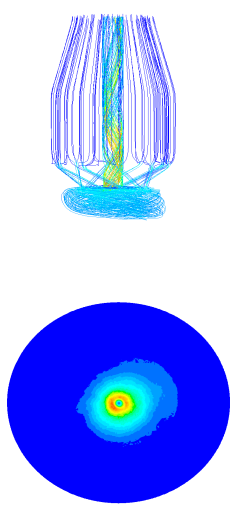

(e) $10 \mathrm{kPa}$ inlet, $-50 \mathrm{kPa}$ outlet
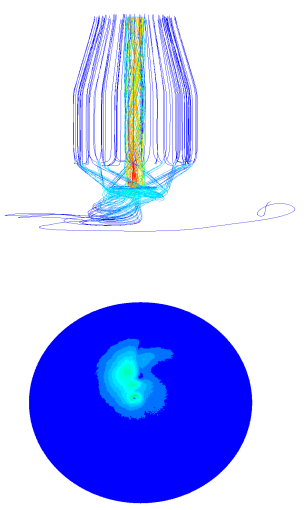

(g) $10 \mathrm{kPa}$ inlet, $-70 \mathrm{kPa}$ outlet
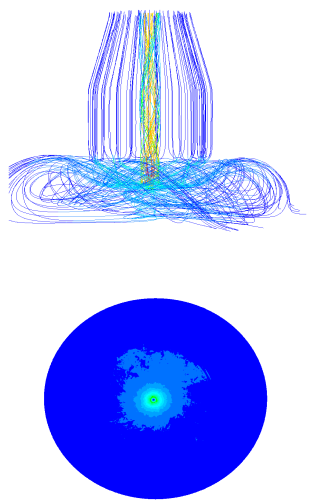

(c) $10 \mathrm{kPa}$ inlet, $-30 \mathrm{kPa}$ outlet
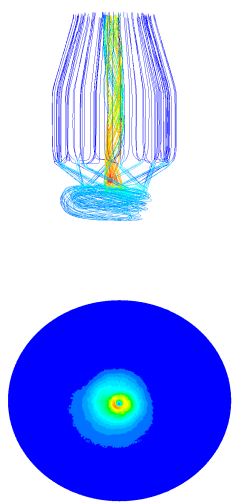

(f) $10 \mathrm{kPa}$ inlet, $-60 \mathrm{kPa}$ outlet

Figure 3.1: Velocity pathlines and corresponding shear stress plots are shown for a fixed geometry of $60^{\circ}$ tangental component and $15^{\circ}$ radial component. Shear stress plots are evaluated at the impinged surface. Pathlines are released from the positive pressure inlet and tracked until they exit the computational domain. 

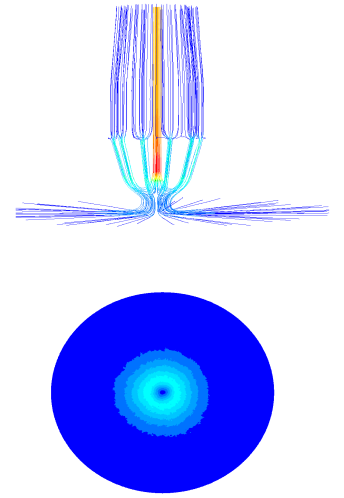

(a) $0^{\circ}$ Tangential, $15^{\circ}$ Radial
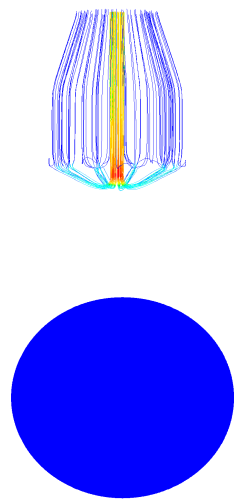

(d) $0^{\circ}$ Tangential, $60^{\circ}$ Radial
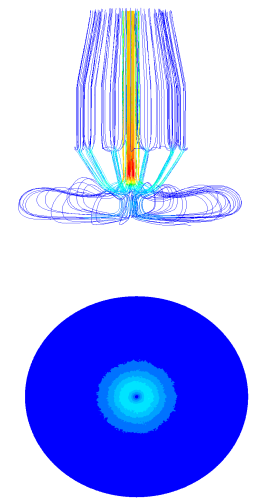

(b) $0^{\circ}$ Tangential, $30^{\circ}$ Radial

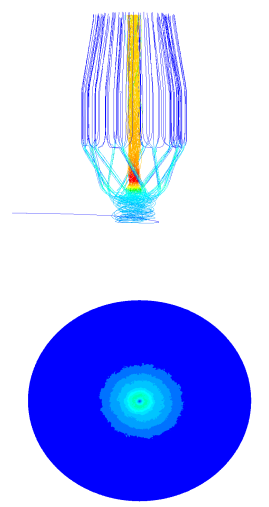

(e) $30^{\circ}$ Tangential, $15^{\circ}$ Radial
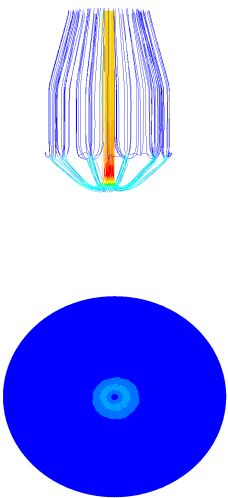

(c) $0^{\circ}$ Tangential, $45^{\circ}$ Radial
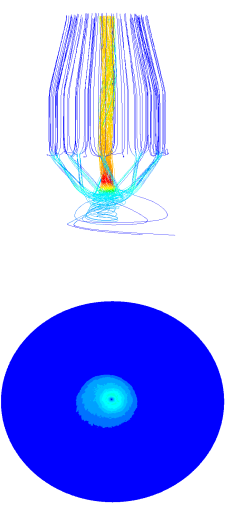

(f) $30^{\circ}$ Tangential, $30^{\circ}$ Radial

Figure 3.2: Velocity pathlines and corresponding shear stress plots are shown for a fixed pressure of $10 \mathrm{kPa}$ inlet and $-40 \mathrm{kPa}$ outlet. Shear stress plots are evaluated at the impinged surface. Pathlines are released from the positive pressure inlet and tracked until they exit the domain. 

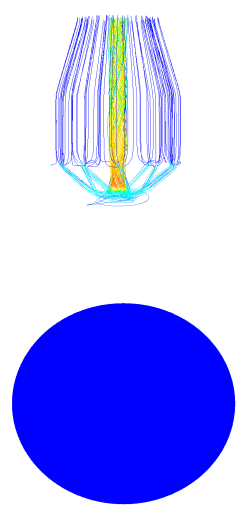

(a) $30^{\circ}$ Tangential, $45^{\circ}$ Radial
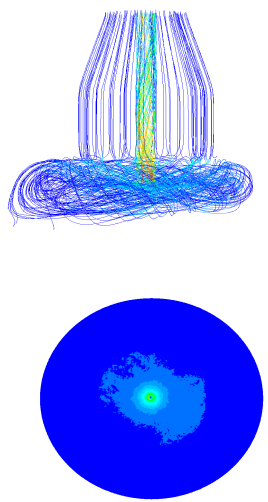
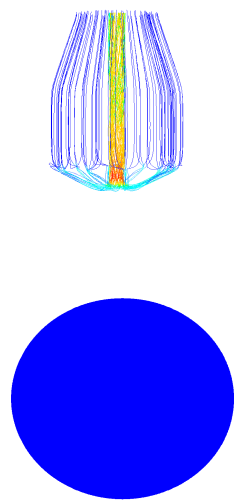

(b) $30^{\circ}$ Tangential, $60^{\circ}$ Radial
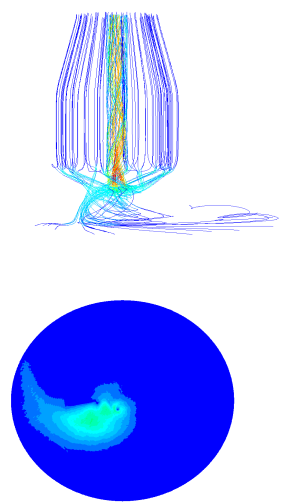
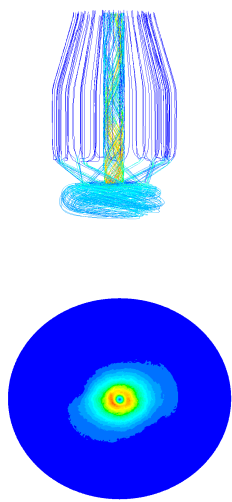

(c) $60^{\circ}$ Tangential, $15^{\circ}$ Radial
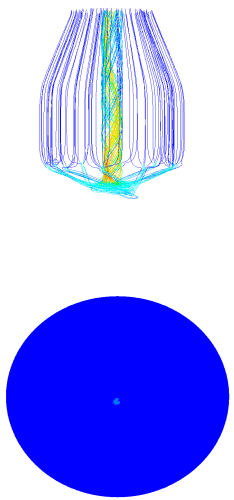

(d) $60^{\circ}$ Tangential, $30^{\circ}$ Radial

(e) $60^{\circ}$ Tangential, $45^{\circ}$ Radial

(f) $60^{\circ}$ Tangential, $60^{\circ}$ Radial

Figure 3.3: Velocity pathlines and corresponding shear stress plots are shown for a fixed pressure of $10 \mathrm{kPa}$ inlet and $-40 \mathrm{kPa}$ outlet. Shear stress plots are evaluated at the impinged surface. Pathlines are released from the positive pressure inlet and tracked until they exit the domain. 


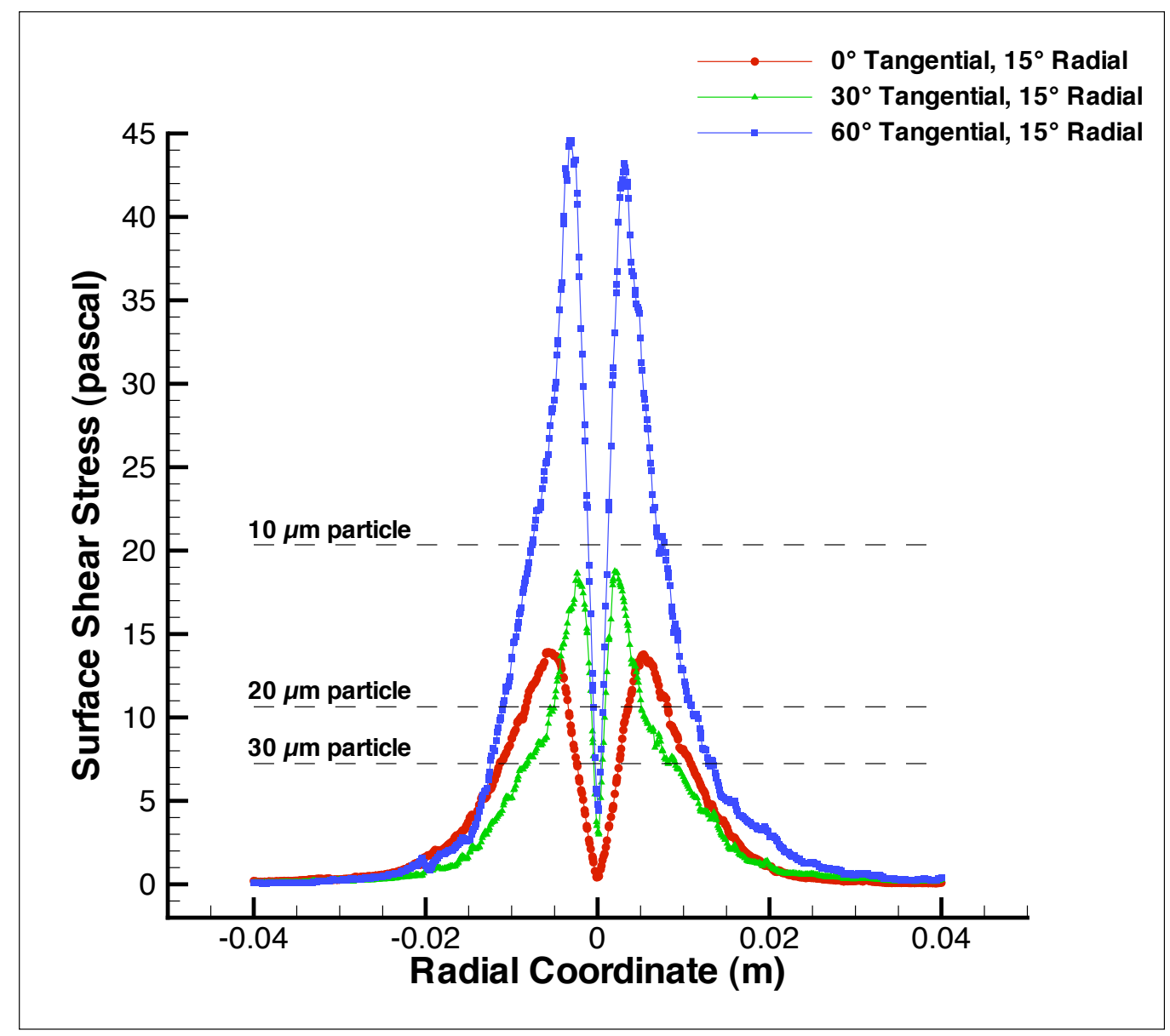

Figure 3.4: A radial distribution of shear stress on the impinged surface is shown for various tangential components and a fixed $15^{\circ}$ radial component. Inlet and outlet pressures are held constant at $10 \mathrm{kPa}$ and $-40 \mathrm{kPa}$ respectively for all combinations. Maximum shear stress is present for a geometry of $60^{\circ}$ tangential component and $15^{\circ}$ radial component. The dashed lines indicate the minimum shear stress required to initiate particle rolling. 


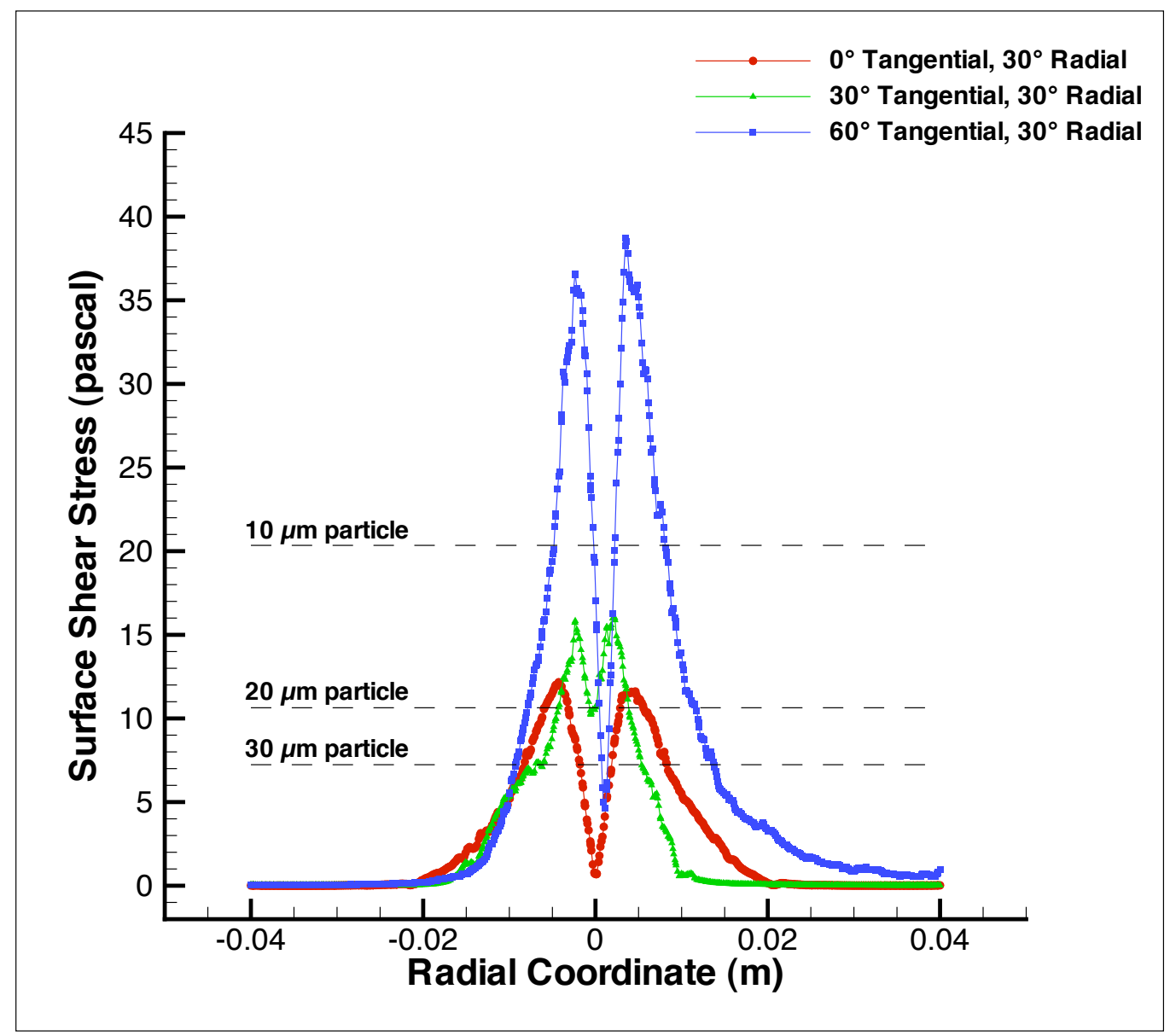

Figure 3.5: A radial distribution of shear stress on the impinged surface is shown for various tangential components and a fixed $30^{\circ}$ radial component. Inlet and outlet pressures are held constant at $10 \mathrm{kPa}$ and $-40 \mathrm{kPa}$ respectively for all combinations. The dashed lines indicate the minimum shear stress required to initiate particle rolling. 


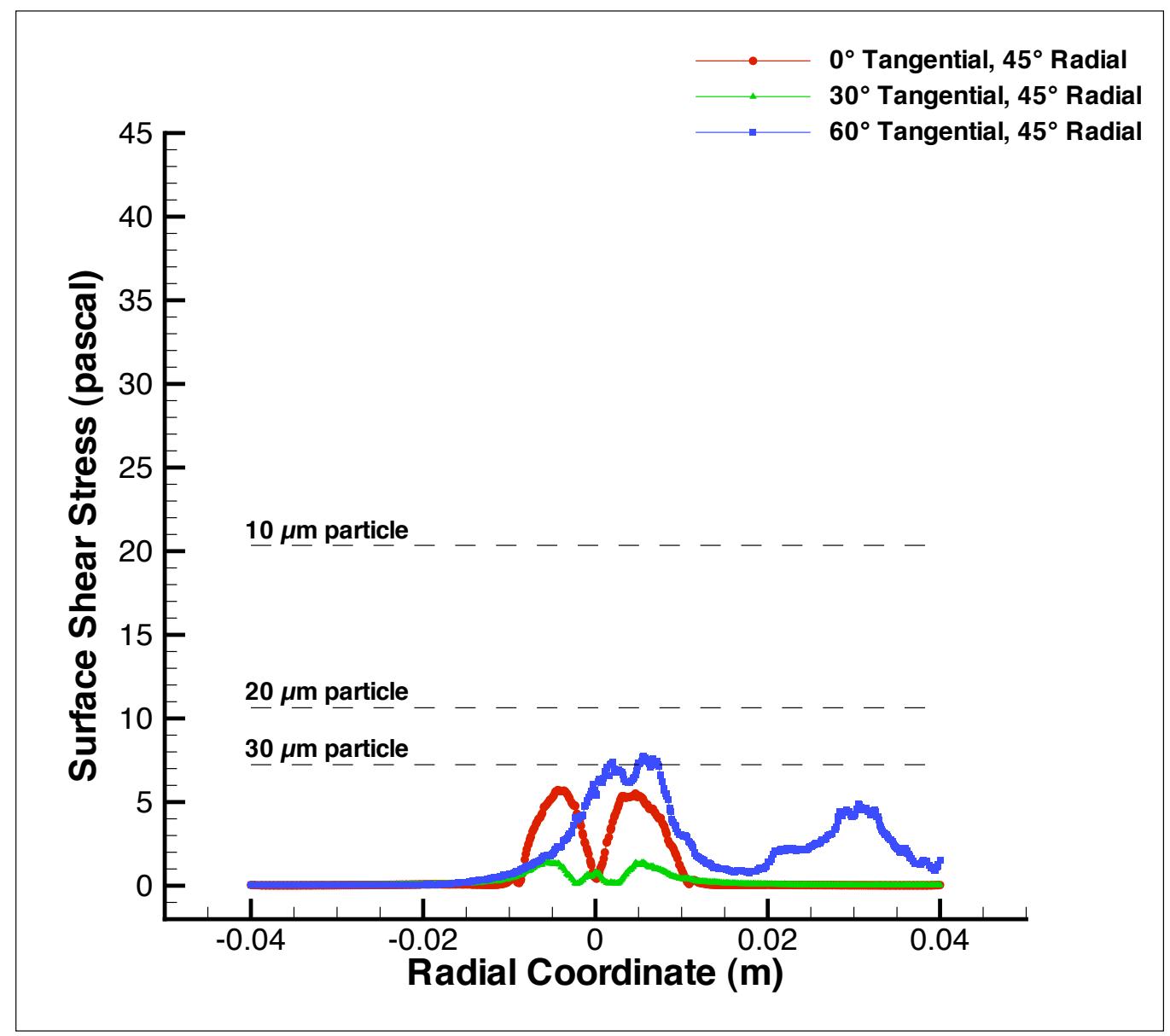

Figure 3.6: A radial distribution of shear stress on the impinged surface is shown for various tangential components and a fixed $45^{\circ}$ radial component. Inlet and outlet pressures are held constant at $10 \mathrm{kPa}$ and $-40 \mathrm{kPa}$ respectively for all combinations. The dashed lines indicate the minimum shear stress required to initiate particle rolling. 


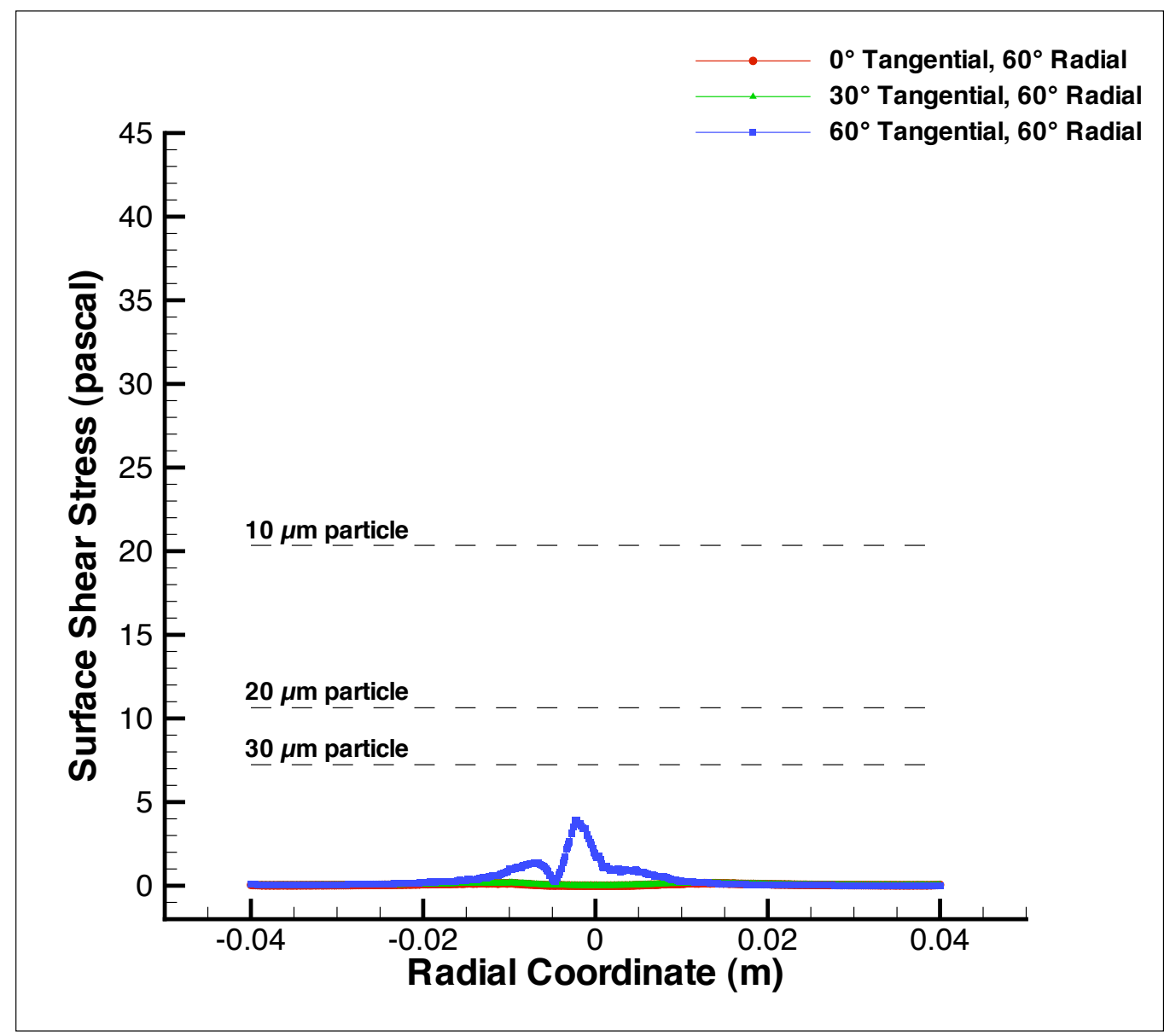

Figure 3.7: A radial distribution of shear stress on the impinged surface is shown for various tangential components and a fixed $60^{\circ}$ radial component. Inlet and outlet pressures are held constant at $10 \mathrm{kPa}$ and $-40 \mathrm{kPa}$ respectively for all combinations. The dashed lines indicate the minimum shear stress required to initiate particle rolling. 


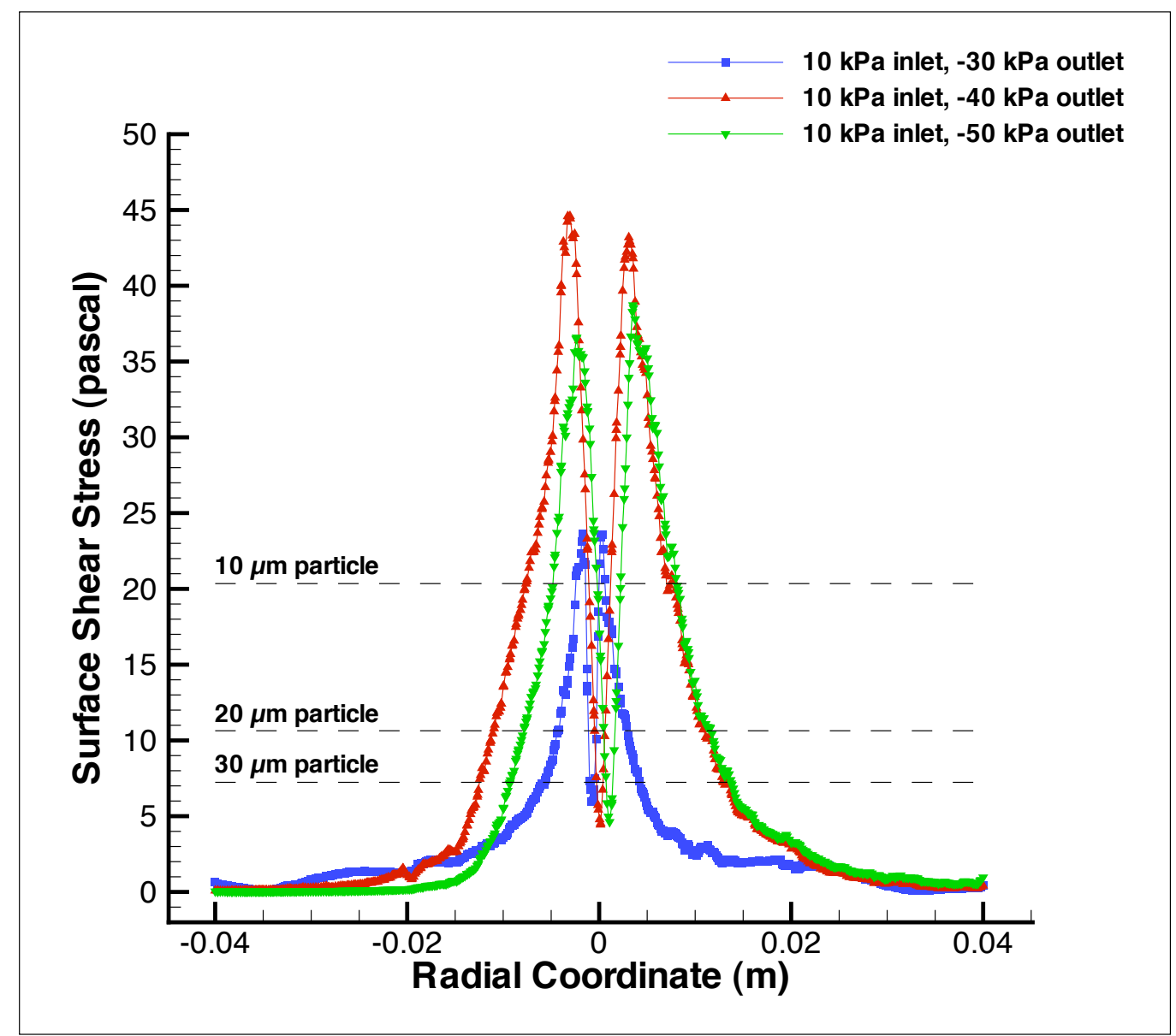

Figure 3.8: A radial distribution of shear stress on the impinged surface is shown for a constant geometry of $60^{\circ}$ tangential component and $15^{\circ}$ radial component. The inlet pressure is held at a constant $10 \mathrm{kPa}$ and the outlet pressure ranges form -30 $\mathrm{kPa}$ to $-50 \mathrm{kPa}$ in increments of $10 \mathrm{kPa}$. Maximum shear stress occurs at a condition of $10 \mathrm{kPa}$ inlet pressure and $-40 \mathrm{kPa}$ outlet pressure. The dashed lines indicate the minimum shear stress required to initiate particle rolling. 


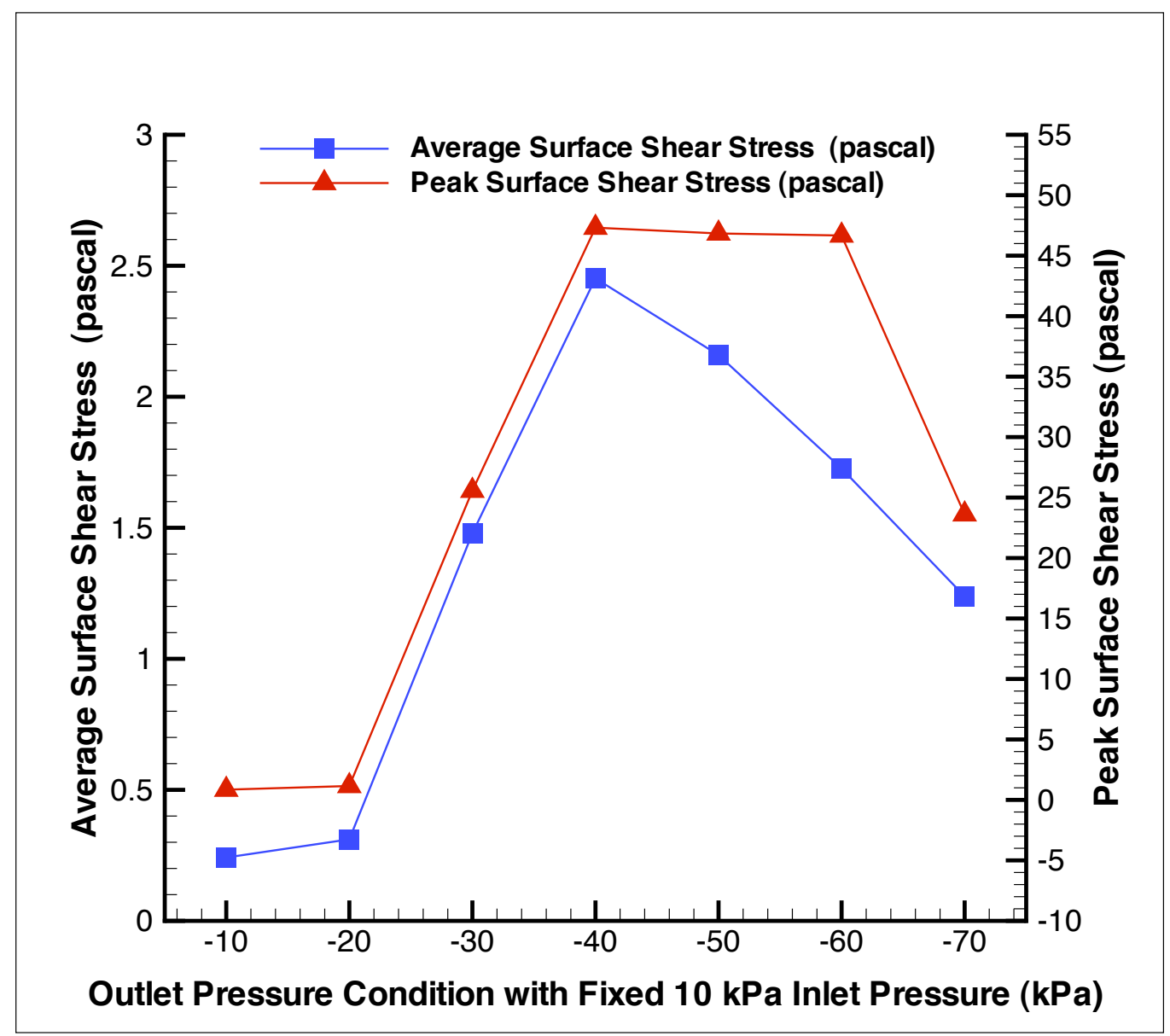

Figure 3.9: Average and peak shear stress on the impinged surface is shown for a constant geometry of $60^{\circ}$ tangential component and $15^{\circ}$ radial component. The inlet pressure is held at a constant $10 \mathrm{kPa}$ and the outlet pressure ranges form $-10 \mathrm{kPa}$ to $-70 \mathrm{kPa}$ in increments of $10 \mathrm{kPa}$. A maximum average shear stress and maximum peak shear stress occurs at a condition of $10 \mathrm{kPa}$ inlet pressure and $-40 \mathrm{kPa}$ outlet pressure. 


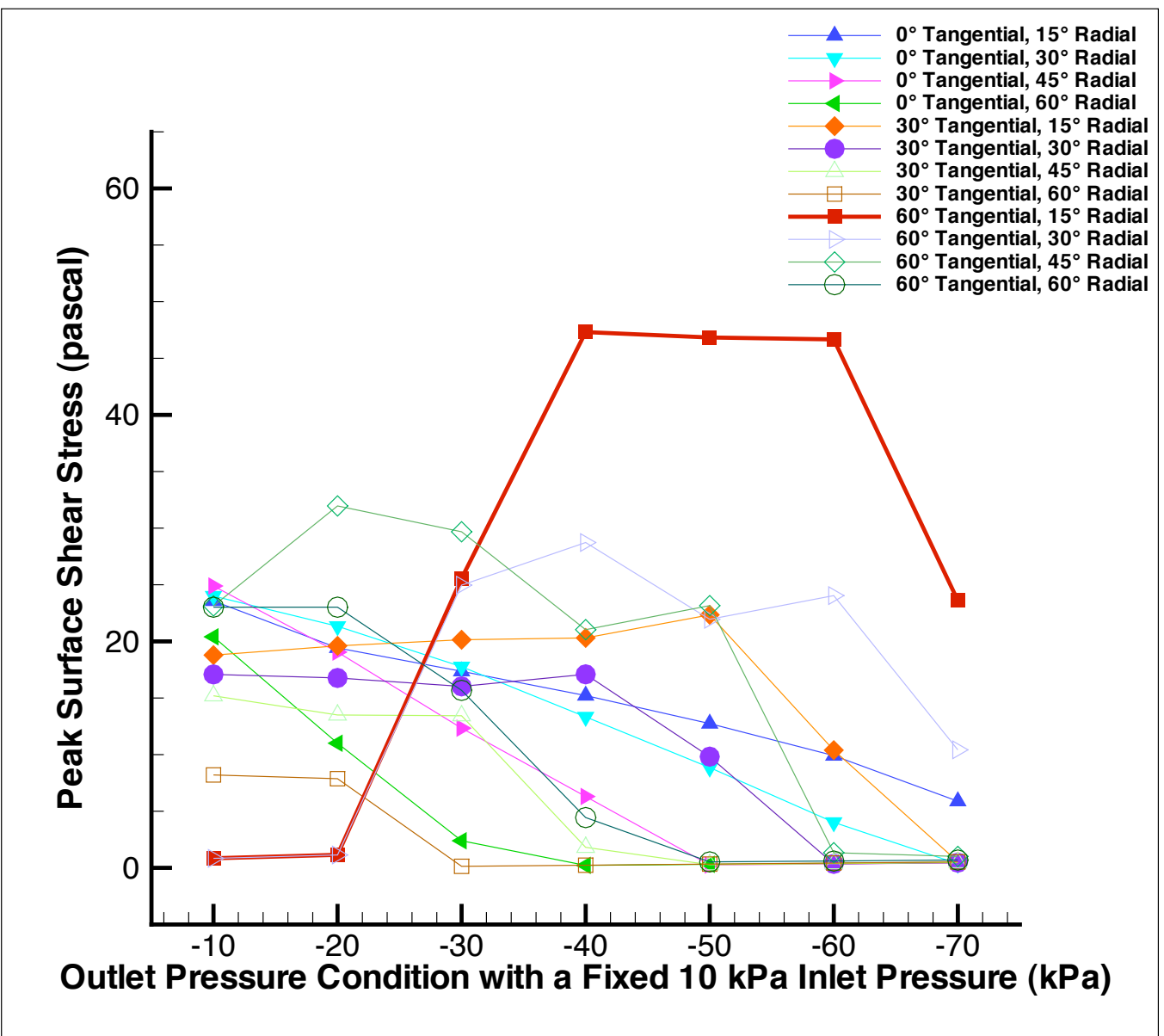

Figure 3.10: All parametric permutations are summarized as a function of peak shear stress. The optimal configuration is shown in bold. 


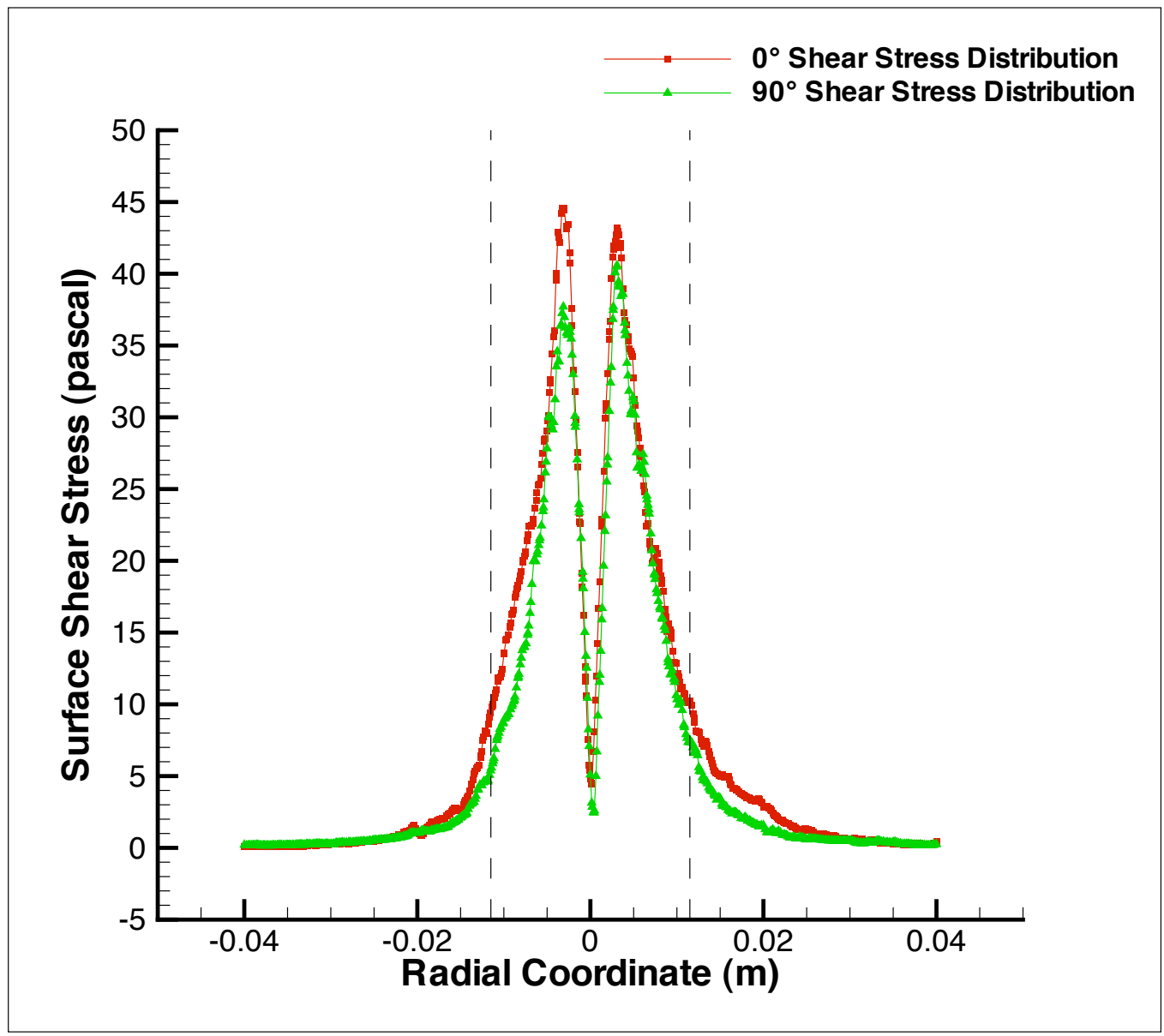

Figure 3.11: Azimuthal variation is shown for two perpendicular radial shear stress distributions. The distributions correspond the the optimal configuration of $60^{\circ}$ tangential component and $15^{\circ}$ radial component. A slight variation can be seen in the peak shear stress values. The dashed lines represent the outer diameter of the nozzle face. 


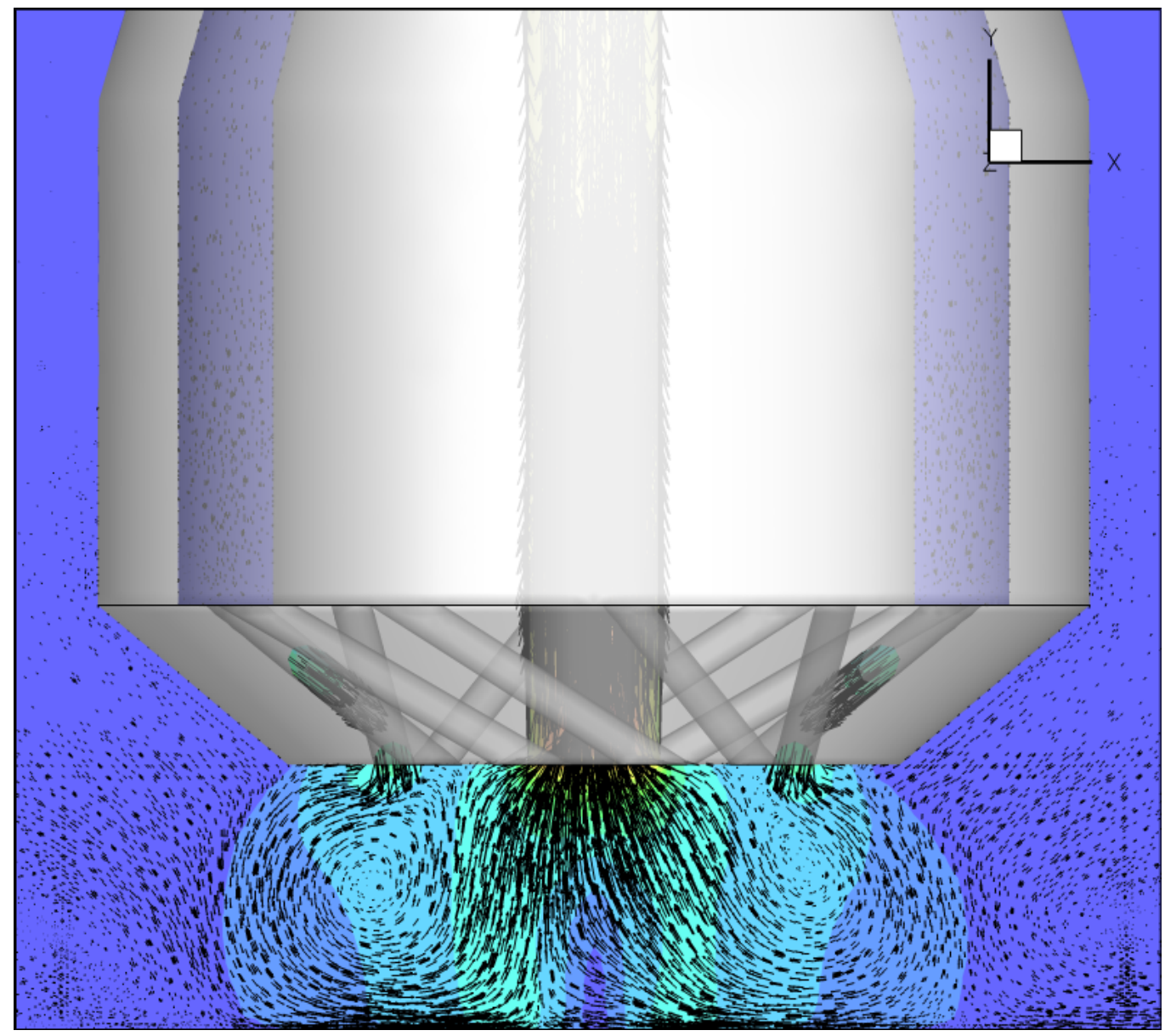

Figure 3.12: Velocity vectors are shown for a geometry of $60^{\circ}$ tangental component and $15^{\circ}$ radial component. The inlet pressure is defined as $10 \mathrm{kPa}$ and outlet pressure is defined as $-40 \mathrm{kPa}$. The background plot is flooded with contours of velocity magnitude. The velocity vectors depict fluid motion from the positive pressure jets to the impinged surface and out through the negative pressure port. 


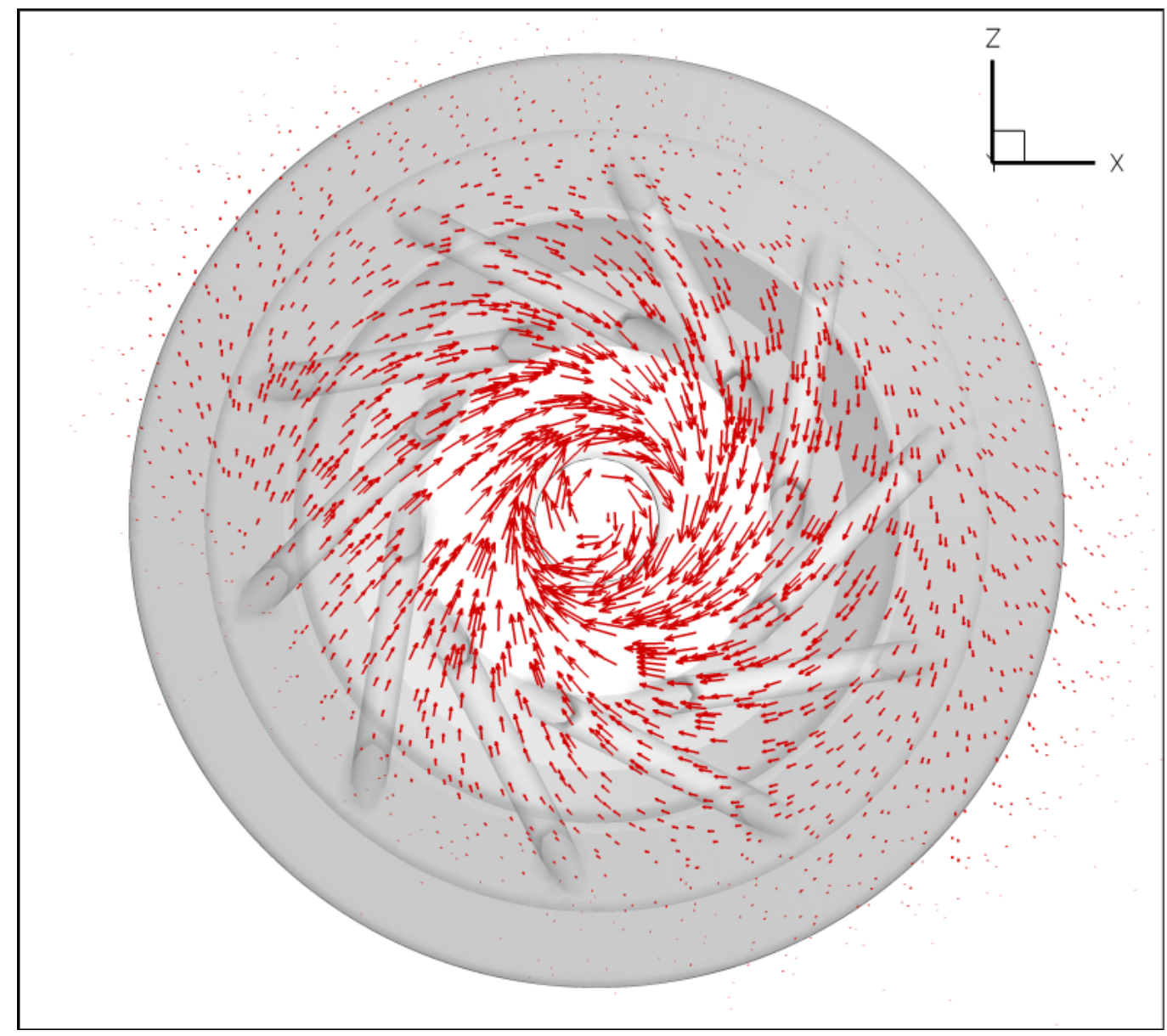

Figure 3.13: Velocity vectors are shown at impinged surface for a geometry of $60^{\circ}$ tangental component and $15^{\circ}$ radial component. The inlet pressure is defined as 10 $\mathrm{kPa}$ and outlet pressure is defined as $-40 \mathrm{kPa}$. A clockwise rotating vortex pattern can be seen centered about the nozzle's negative pressure port. 


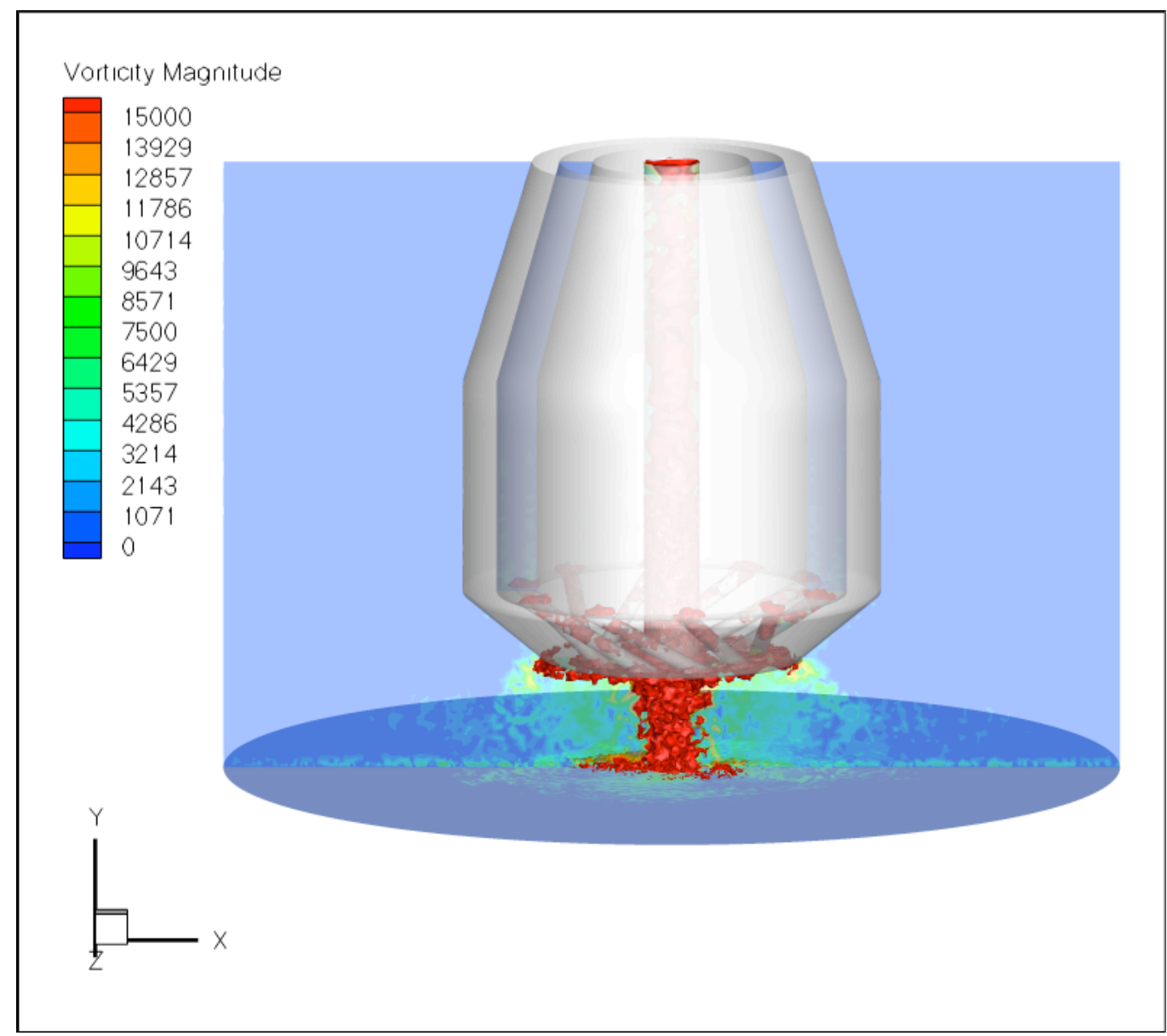

Figure 3.14: An iso-surface of vorticity is shown for a geometry of $60^{\circ}$ tangental component and $15^{\circ}$ radial component. The inlet pressure is defined as $10 \mathrm{kPa}$ and outlet pressure is defined as $-40 \mathrm{kPa}$. The iso-surface is drawn at a vorticity magnitude of 15,000 . Contour plots of vorticity are depicted on the impinged surface and a vertical plane intersecting the axis of rotation. 


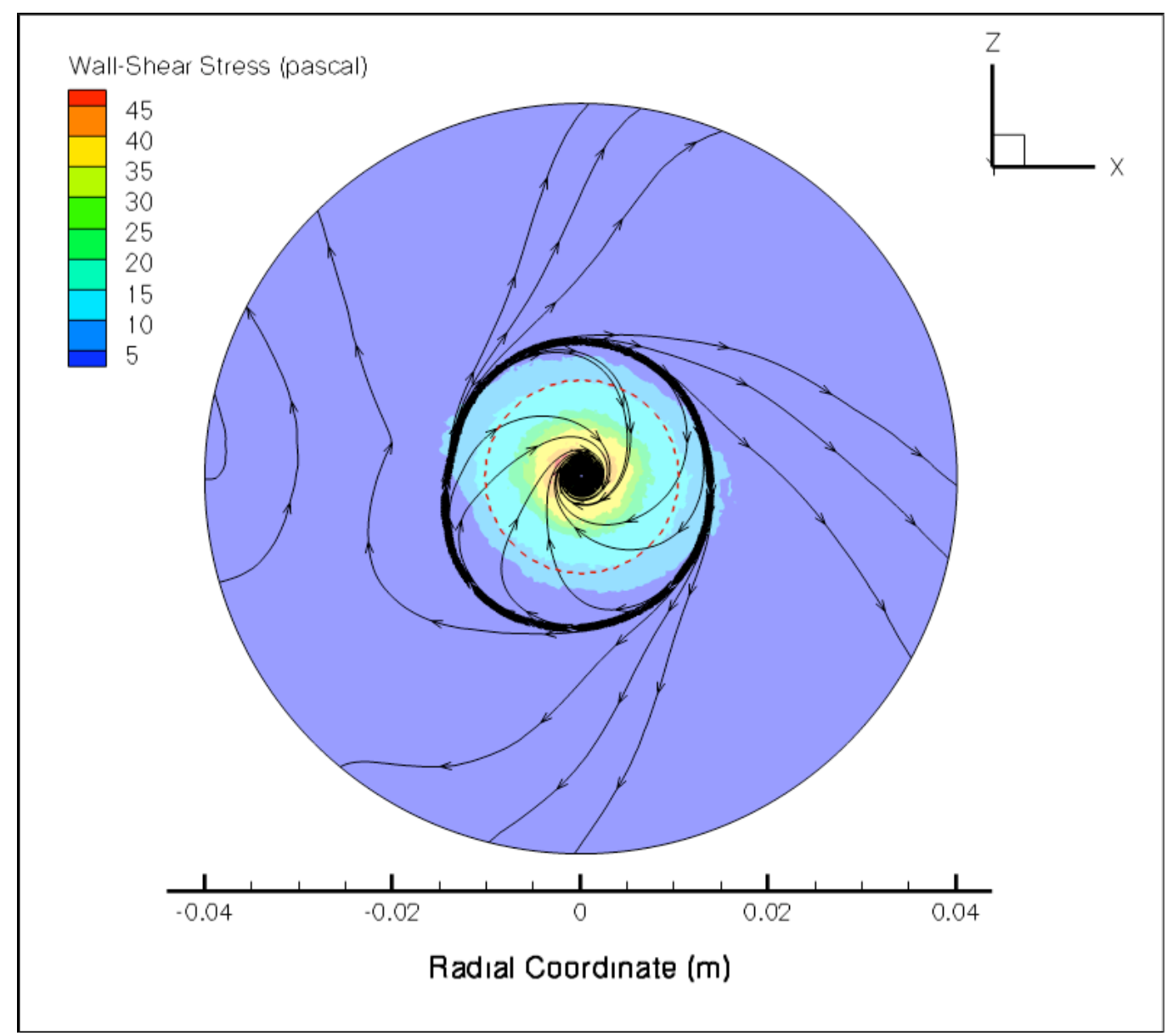

Figure 3.15: Stress lines are shown along the impinged surface for a geometry of $60^{\circ}$ tangental component and $15^{\circ}$ radial component. The inlet pressure is defined as $10 \mathrm{kPa}$ and outlet pressure is defined as $-40 \mathrm{kPa}$. A contour plot of wall shear stress is depicted on the impinged surface. Nearly all enhanced surface shear stress is contained within the annular transition region. The dashed red line represents the circumference of the nozzle tip. 
Table 3.3: Pressure conditions and corresponding Reynolds numbers are shown for a fixed pressure ratio and varying pressure magnitude. Reynolds numbers were calculated for the positive pressure jets based on a diameter of $1.5 \mathrm{~mm}$.

\begin{tabular}{|c|c|c|}
\hline \multicolumn{3}{|c|}{ Pressure Magnitude Study } \\
\hline Inlet Pressure $(\mathrm{kPa})$ & Outlet Pressure $(\mathrm{kPa})$ & Reynolds Number \\
\hline 1 & 4 & 2581 \\
\hline 2 & 8 & 3137 \\
\hline 3 & 12 & 4477 \\
\hline 4 & 16 & 5162 \\
\hline 5 & 20 & 5778 \\
\hline 6 & 24 & 6334 \\
\hline 7 & 28 & 6840 \\
\hline 8 & 32 & 7306 \\
\hline 9 & 36 & 7733 \\
\hline 10 & 40 & 8190 \\
\hline
\end{tabular}




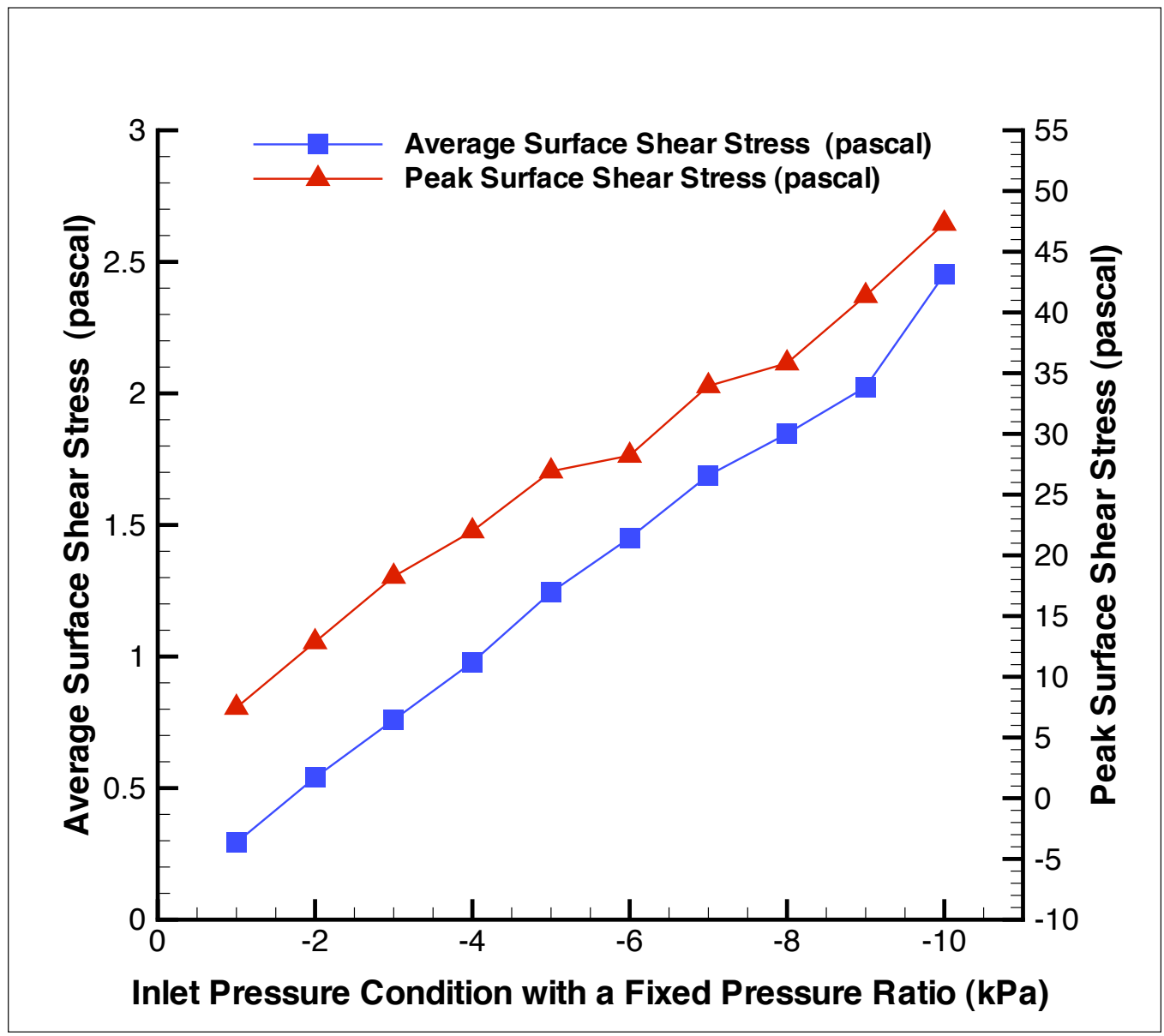

Figure 3.16: A plot of surface shear stress is shown for a geometry of $60^{\circ}$ tangental component and $15^{\circ}$ radial component. The applied pressure was fixed at a 1:4 inlet to outlet ratio. The inlet pressure varied from $1 \mathrm{kPa}$ to $10 \mathrm{kPa}$ while the outlet pressure was adjusted to maintain the desired 1:4 ratio. 


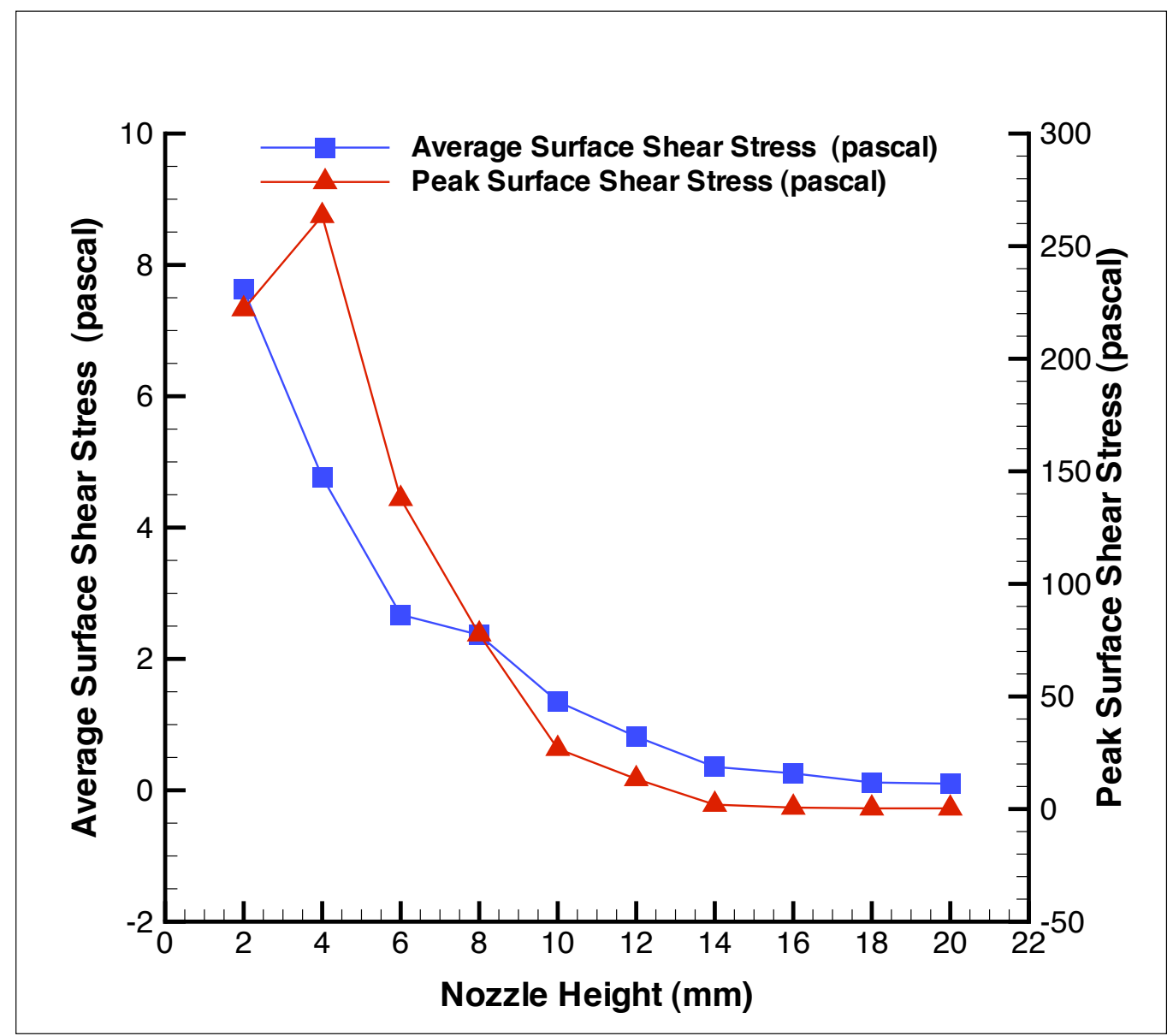

Figure 3.17: A plot of surface shear stress is shown for varying nozzle heights. The applied inlet pressure is prescribed at $10 \mathrm{kPa}$ and outlet pressure is defined as -40 $\mathrm{kPa}$. A nonlinear relation between nozzle heigh and surface shear stress is shown. 

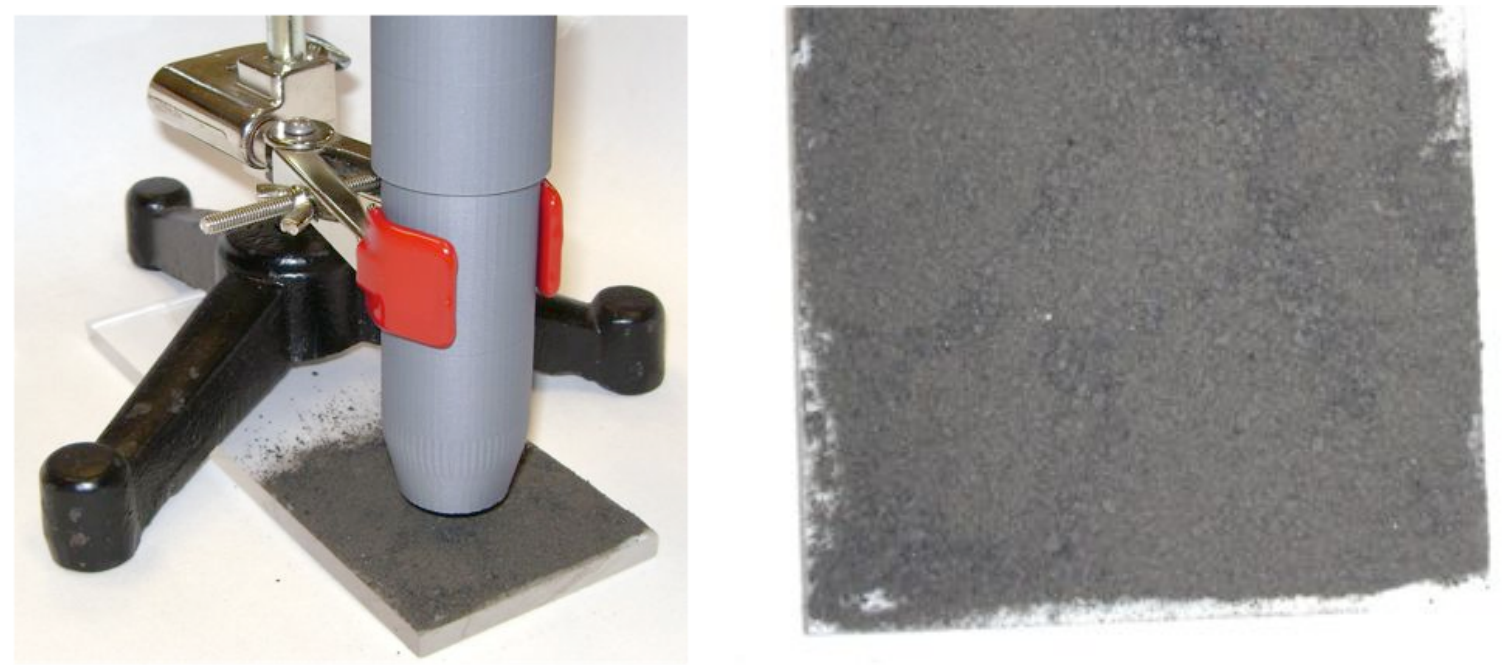

Figure 3.18: Left: The experimental apparatus and nozzle are suspended $10 \mathrm{~mm}$ above an acrylic substrate evenly coated with Lunar simulant (JSC-II). Right: The acrylic substrate and Lunar simulant after only negative pressure is applied to the nozzle's central port.
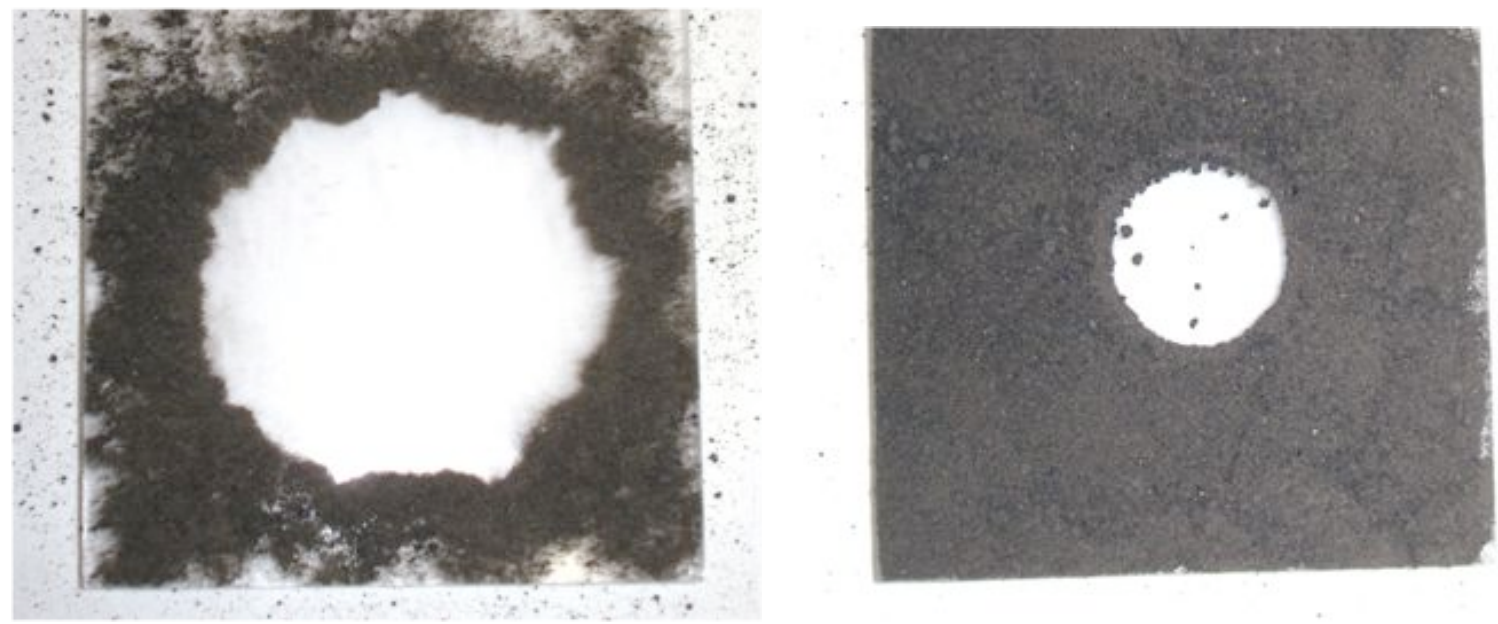

Figure 3.19: Left: The acrylic substrate and Lunar simulant after only positive pressure is applied to the ring of outer jets. Right: The acrylic substrate and Lunar simulant after positive and negative pressure is simultaneously applied to the ring of outer jets and central port respectively. 

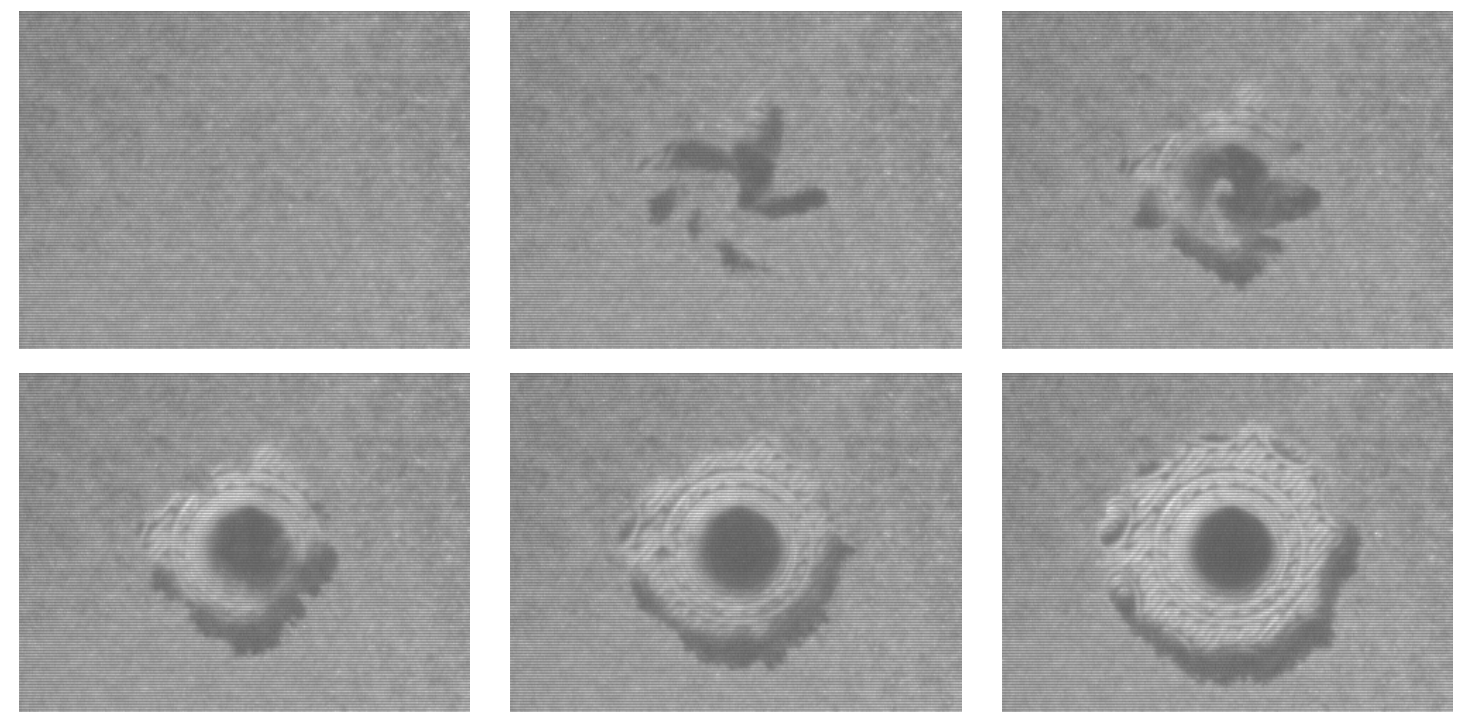

Figure 3.20: A progressive set of photographs depicting the particle removal process is shown. The impingement surface is imaged from the underside of a transparent impingement surface. Initially the surface is evenly covered with a regolith simulant. As negative and positive pressures are applied, the particles are dislodged and removed from the impingement surface. As particles are removed, the surface of the nozzle and ports become visible. 

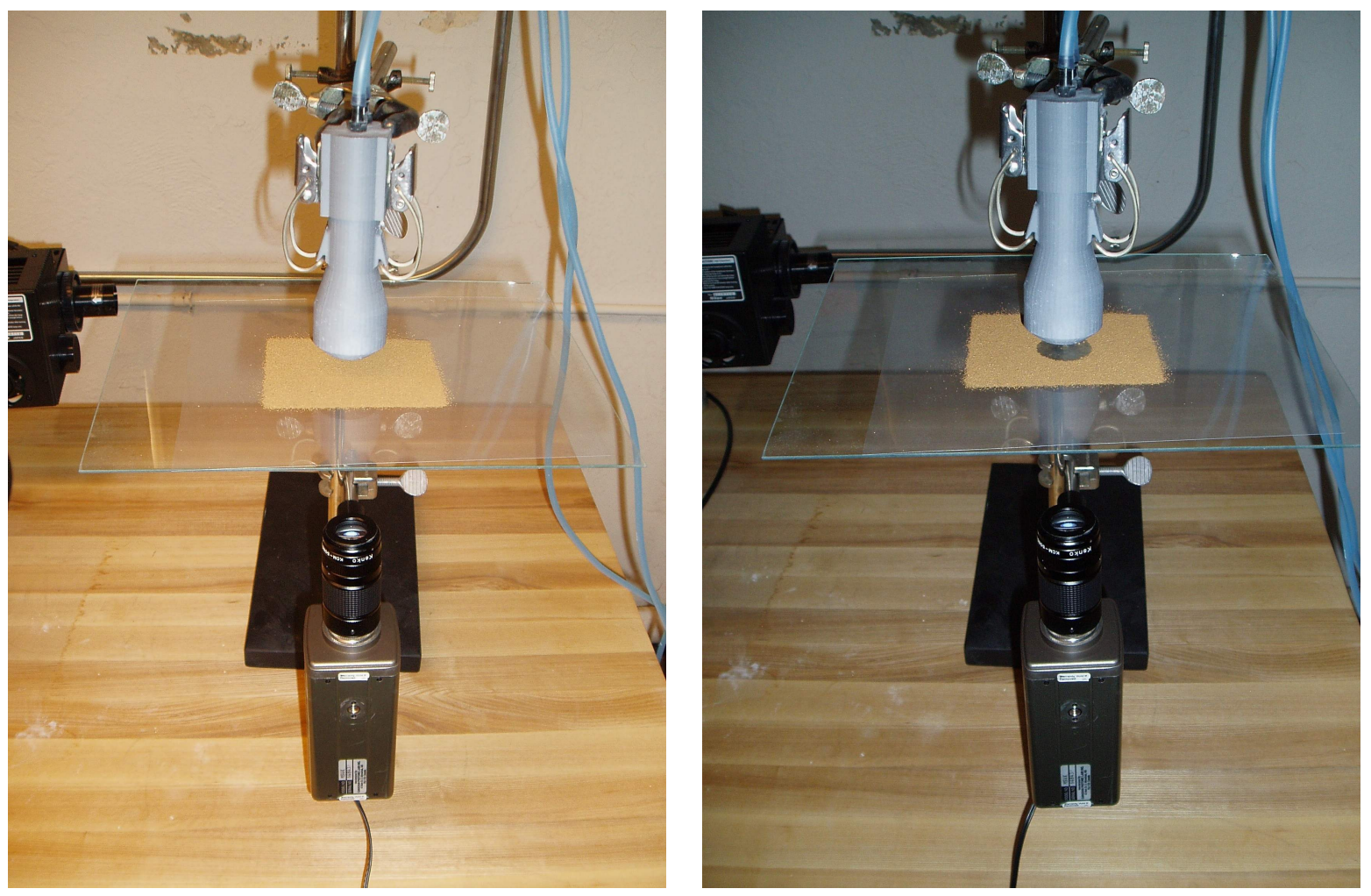

Figure 3.21: The experimental high-speed photography apparatus consists of a prototype nozzle positioned above a glass impingement surface. The removal process is imaged from beneath the impingement surface using a high-speed camera. The left image shows the surface before particle removal. The right image illustrates the surface after the removal process. 


\section{Chapter 4}

\section{Estimation of Micro-Particle}

\section{Removal Efficiency}

The removal process of an adhered particle consists of two essential stages. First, threshold shear conditions must exist such that the initially stationary particle is freed from the surface and begins to roll and dynamically interact with other par-

ticles, as described by Schmeeckle et al. (2003) [24]. Once in motion, the moving particle is lifted from the surface and transported away by aerodynamic drag forces. Previous studies have indicated that of these two stages, 'rolling' and 'lift-off', it is the initiation of rolling that establishes the critical flow conditions for particle removal. Put another way, flow conditions sufficient to initiate rolling will also prove sufficient for aerodynamic lift-off. Thus, to estimate the particle removal efficiency of the bound-vortex flow one must compare the expected wall shear stress distribution with critical shear stress levels for initiation of the adhered particle rolling. 


\subsection{Derivation of Critical Shear Condition for Par- ticle Removal}

To develop the critical flow condition, consider a stationary, spherical particle adhered to the surface solely by van der Waal's force. Dynamically, a stationary particle will begin rolling when there is a non-zero torque about contact point with the surface. There are two essential sources of torque present: (1) an aerodynamic torque arising from the drag force acting through the particle centroid; and (2) an adhesive torque arising from surface contact forces. The aerodynamic drag force for micro-particles can be predicted in terms of a modified Stokes drag law of the form

$$
F_{D}=6 \pi \mu R U f
$$

where $\mu$ is the fluid dynamic viscosity, $R$ is the particle radius, $U$ is the fluid velocity at the particle centroid and $f$ is a correction factor that accounts for wall effects. It is convenient to write $U$ in terms of a shear rate $\dot{\gamma}$ as

$$
U=\dot{\gamma} R
$$

The correction factor is known to be dependent on the Reynolds number (based on

the particle size); a classical correlation is given by Schiller and Naumann (1933) [23] as

$$
f=1+0.15 R e_{P}^{0.687}
$$

and a more recent empirical formulation based on numerical simulations by Sweeney and Finlay (2007) [26] is given as

$$
f=1.7005\left[1-\frac{0.2817}{R e_{P}^{0.0826}} \sin ^{-1}\left(0.238 R e_{P}\right)\right]^{-1}
$$

Opposing this aerodynamic torque is a resistive torque linked to the surface adhesion which arises when a particle first begins to roll. The adhesive torque $M_{A}$ takes 
the form (Dominik and Tielens, 1995) [8]

$$
M_{A}=-k_{R} R \theta,
$$

Here $k_{R}$ is a resistive rolling coefficient and $R \theta$ represents a rolling displacement. The coefficient $k_{R}$ is a complicated quantity that depends on a combination of parameters including the contact region radius for the particle, the particle's elastic modulus and the adhesive surface energy density $\sigma$. Experimentally, it has been found that adhesive torque reaches a maximum, critical value that can alternatively be represented by a 'critical rolling angle' $\theta_{\text {crit }}$; for micro-sized particles these values have been experimentally characterized by Ding et al. (2008) [7] and found to be on the order of tens of milli-radians.

It follows that rolling will be initiated when the aerodynamic torque exceeds the critical adhesive torque, that is, when $F_{D}>k_{R} \theta_{\text {crit }}$. Using the relations given above and the known expression for $k_{R}$, this rolling condition can be estimated in the following form for the wall shear stress:

$$
\tau_{w}^{c r i t} \simeq \frac{2 \sigma \theta_{\text {crit }}}{f R}
$$

In deriving this result, the simplifying approximation has been made that the aerodynamic lift essentially negates the particle weight. Noting that the correction factor $f$ and the shear stress $\tau_{w}$ both depend on the Reynolds number, it follows that this equation must be solved numerically.

\subsection{Critical Shear Values for Micro-Particles}

Equation (4.6) can be solved with knowledge of the critical rolling angle and the adhesive surface energy. The studies by Ding et al. (2008) [7] and Benz et al. (2006) 
[4] have been used to approximate $\theta_{\text {crit }} \approx 0.04$ mrads and $\sigma \approx 60 \mathrm{~mJ} / \mathrm{m}^{2}$ with the understanding that these parameter estimates are subject to significant uncertainty. Table 4.1 lists critical shear stress for particle sizes ranging from $10 \mu m$ to $100 \mu m$. Shear stress values as a function of particle size can be viewed in a plot form in Figure 4.1. The minimum shear stress required for particle rolling decreases rapidly as particle size increases. With the mean fluid velocity acting on the centroid of the particle, a larger particle offers a greater moment arm. Thus, providing a lower value of critical shear stress.

Critical shear stress values can be used to predict regions of particle removal on the impinged surface. Critical shear stress values for $10 \mu m, 20 \mu m$, and $30 \mu m$ particles have been overlaid in Figures 3.4, 3.5, 3.6, 3.7, and Figure 3.8. Sections where surface shear stress exceeds the critical value, represented by the dashed lines, will provide sufficient shear stress to initiate rolling and eventually liftoff. For most nozzle configurations, an annular region of removal below the nozzle tip is predicted.

JSC-1A lunar mare regolith simulant contains a broad range of particle sizes which can be viewed in Figure 4.2. The particles were measured by NASA using a laser diffraction particle size analyzer [19]. The average particle diameter was determined to be $24.89 \mu \mathrm{m}$ with a median particle diameter of $23.72 \mu \mathrm{m}$. A critical shear stress of 8.9 Pascal was calculated using the average particle diameter of $24.89 \mu \mathrm{m}$. The critical shear stress was then compared with the radial shear stress distribution for the optimal nozzle configuration to determine a predicted removal radius of $0.012 \mathrm{~m}$. The experimental radius was measured to be $0.009 \mathrm{~m}$. A radial shear stress distribution with an overlay of critical shear stress can be seen in Figure 4.3. A graphical comparison between the predicted removal radius and observed experimental removal radius can be seen in Figure 4.4. The larger predicted removal radius could be the result of irregular particle shapes which were not included in the analytical calculation. 
Table 4.1: Critical shear stress is shown as a function of particle size.

\begin{tabular}{|c||c|}
\hline Critical Shear Stress as a Function of Particle Size \\
\hline Particle Size $(\mu m)$ & Critical Shear Stress $(P a)$ \\
\hline 10 & 20.3 \\
20 & 10.6 \\
30 & 7.2 \\
40 & 5.5 \\
50 & 4.4 \\
60 & 3.7 \\
70 & 3.2 \\
80 & 2.8 \\
90 & 2.5 \\
\hline
\end{tabular}




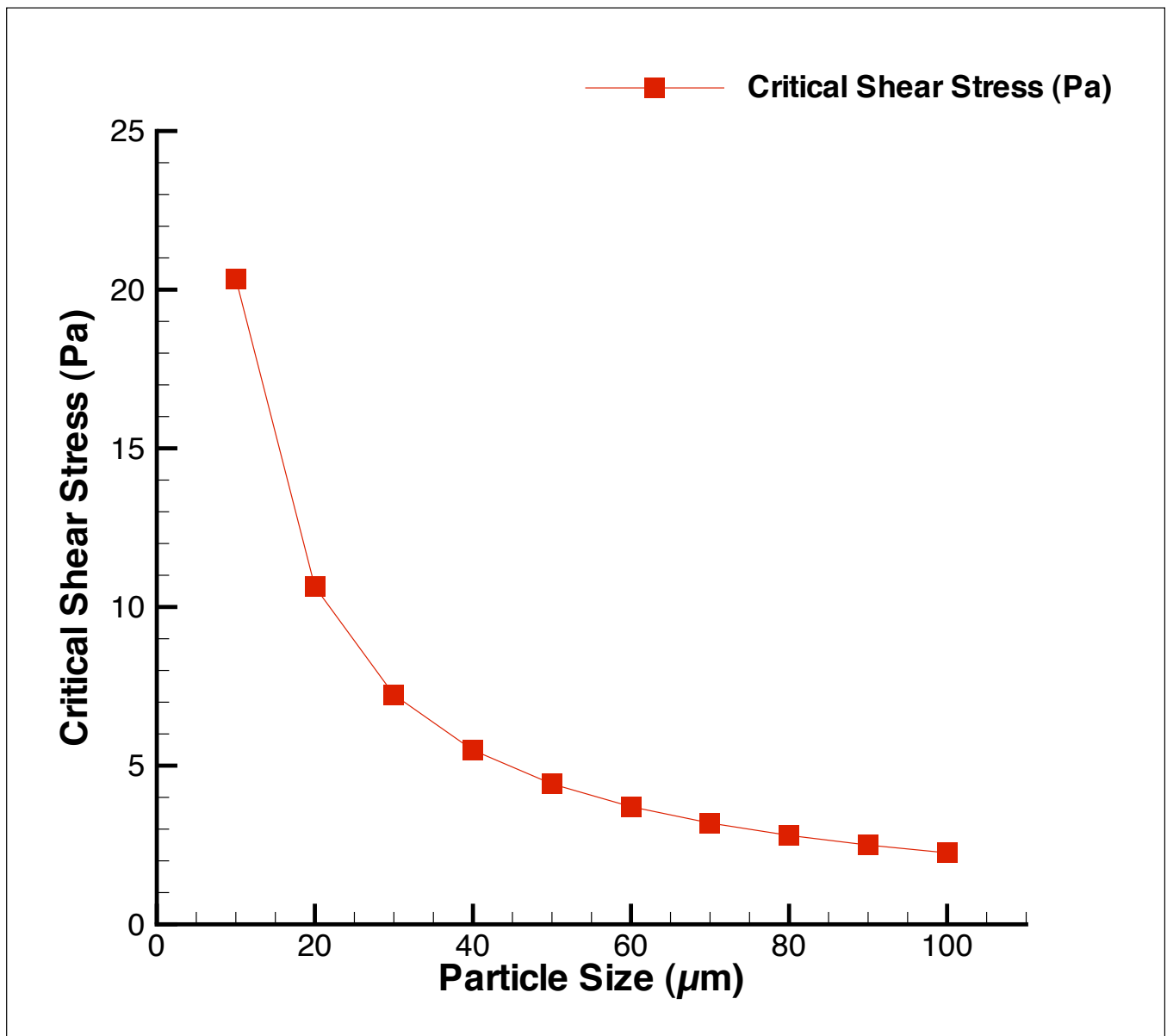

Figure 4.1: Critical shear stress is shown as a function of particle size. Particle size ranges from 10 micron to 100 micron. Critical shear stress scales nonlinearly with particle size. 


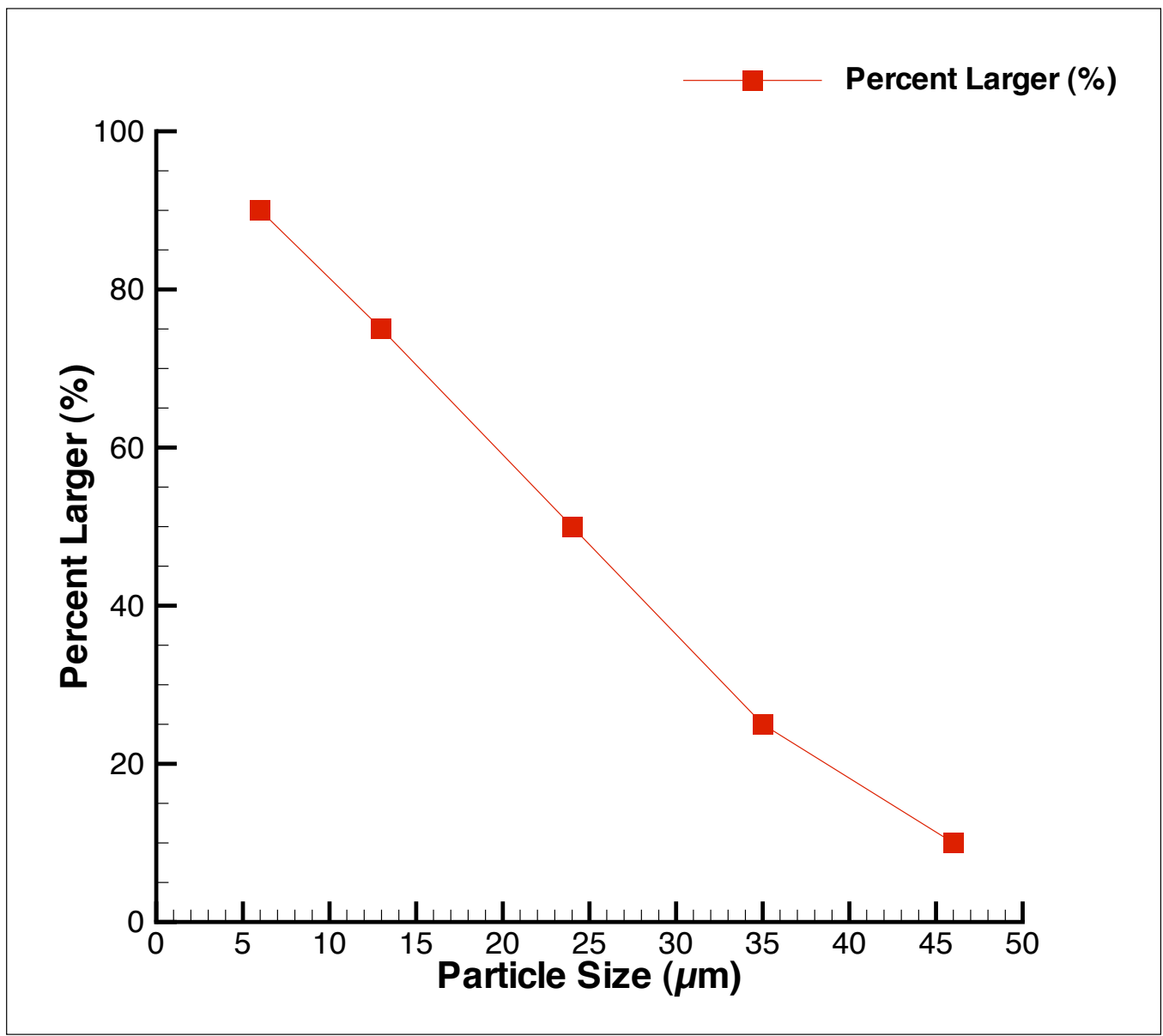

Figure 4.2: A particle size distribution is shown for Lunar simulant JSC-1A. The distribution was calculated by NASA using a laser diffraction particle size analyzer [19]. 


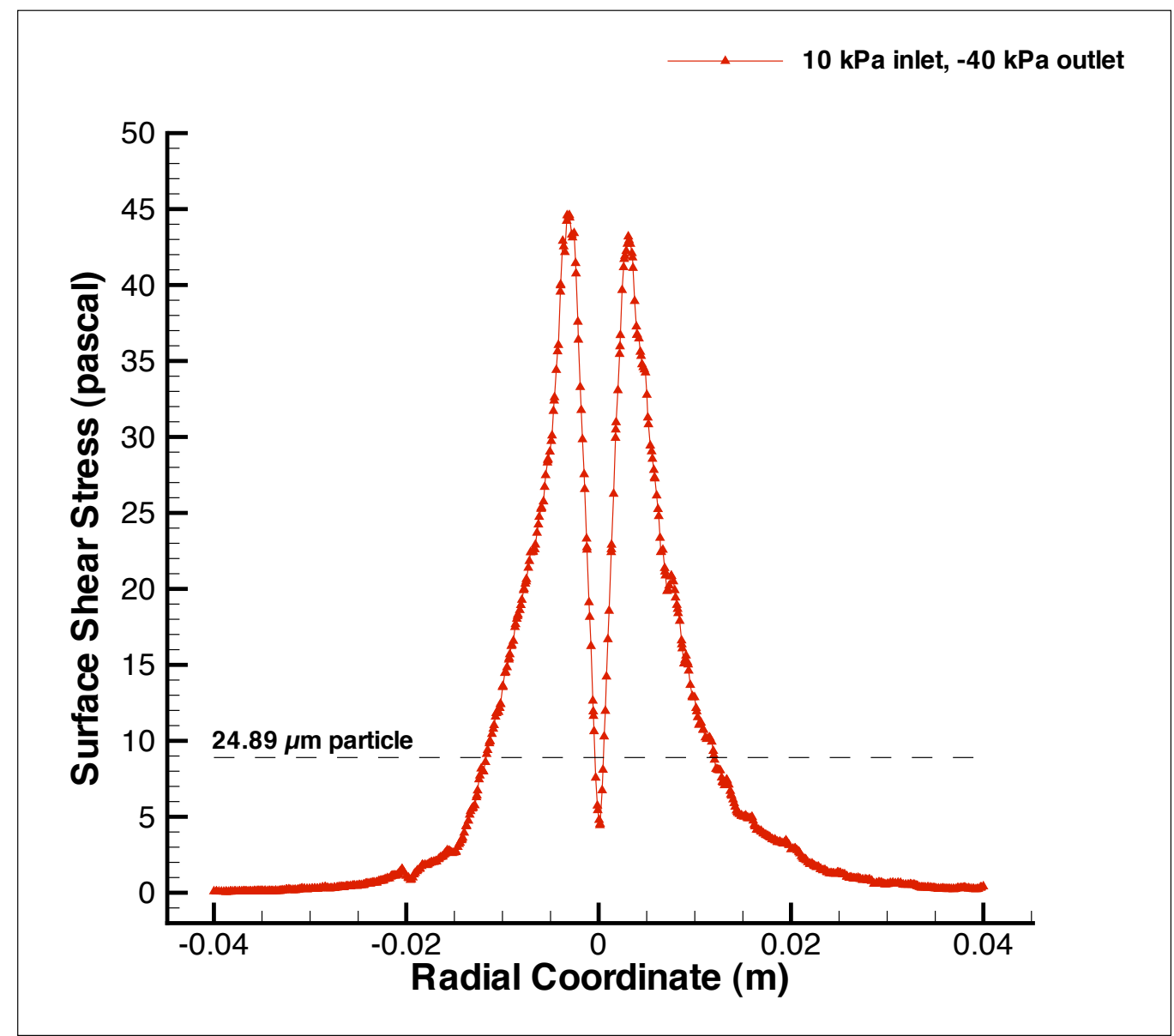

Figure 4.3: A radial shear stress distribution for the optimal nozzle configuration and pressure condition is shown with an overlay of critical shear stress. 


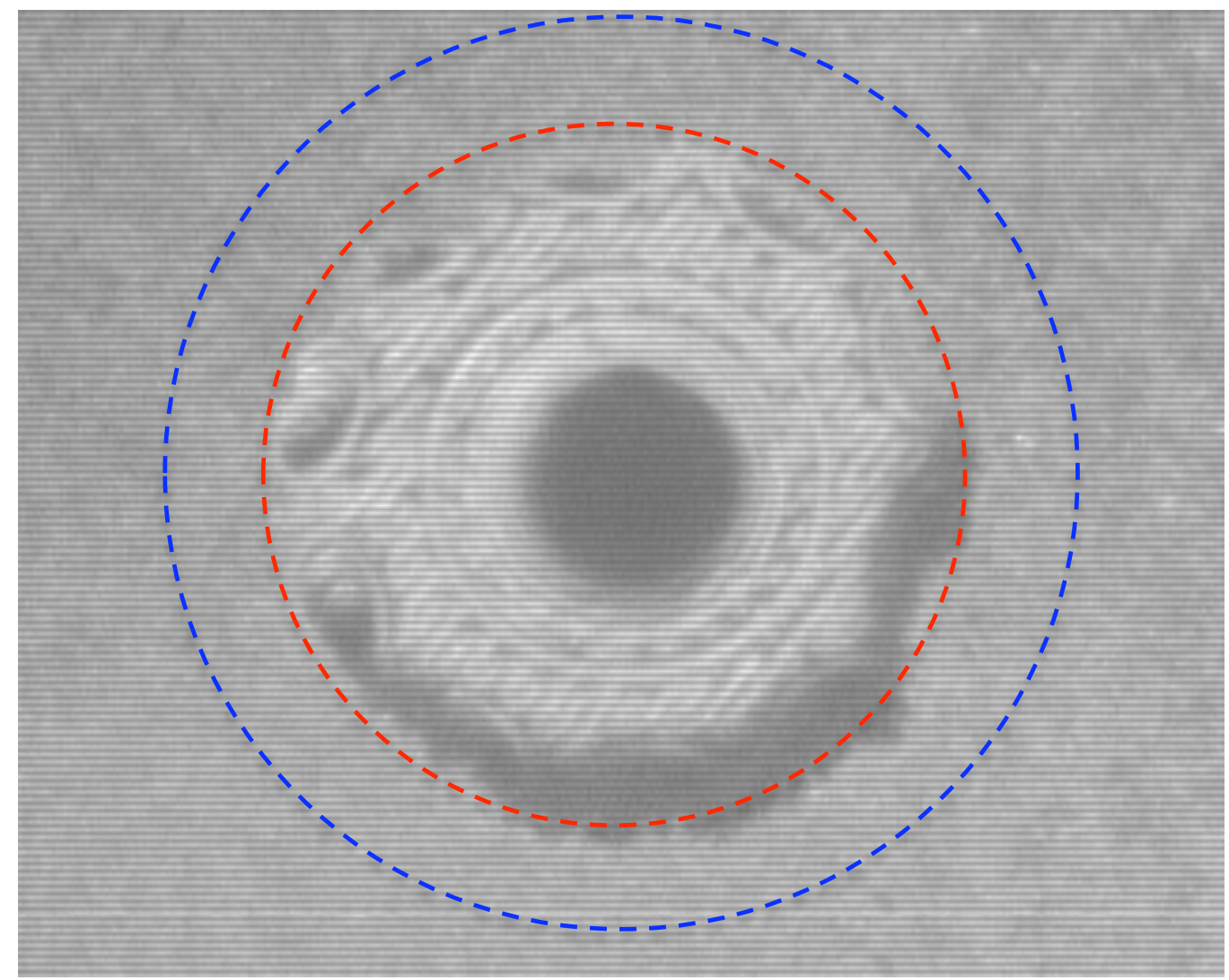

Figure 4.4: A comparison between the experimental removal radius and the predicted radius is shown. The experimental radius is shown in red and the predicted radius is shown in blue. 


\section{Chapter 5}

\section{Conclusions}

In this work we have introduced the concept of an impinging, bound vortex flow structure as a means for removing dust particles from a surface in a controlled manner. The motivating application for this methodology is the need for removing microscopic dust particles from surfaces in lunar and Martian environments. To create the desired flow structure, a composite nozzle device has been designed featuring a ring of angled positive-pressure jets surrounding negative-pressure, central core. The positive pressure jets are defined by a radial and tangential component. Through specific nozzle configurations and pressure conditions, the combination of positive pressure jets and negative pressure core can create a bound vortex flow condition where shear stress on the impinged surface is enhanced. The rotational strength of the vortex was found to increase with an increase in the tangential jet component. The radial component governed the depth of vortex penetration. As the radial component increased, the vortex deviated less from the nozzle's exit plane.

A detailed parametric study of the turbulent 3-D flow structures resulting from varying pressure conditions and nozzle configurations has been performed and utilized to identify design conditions yielding the maximum shear stresses on the impinged 
surface for purposes of dust removal. An 'optimal' nozzle configuration of $60^{\circ}$ tangential component and $15^{\circ}$ radial component was determined to create the greatest shear stress on the impingement surface. A pressure condition of $10 \mathrm{kPa}$ inlet and $-40 \mathrm{kPa}$ outlet was determined to provide the greatest shear stress enhancement. The surface shear stress distribution for the 'optimal' design is largely axisymmetric and is characterized by sharp peaks slightly offset from the central core that quickly decay towards zero at the edge of the nozzle device. Supporting flow visualization experiments with uncharged lunar regolith simulant have demonstrated the efficacy of this methodology: dust particles are efficiently and controllably removed beneath the device leaving other dust particles essentially unperturbed. In summary, the results of this study are encouraging and provide support for the continued development of this concept.

\section{$5.1 \quad$ Future Work}

The integration of acoustic radiation would be a logical progression to further enhance surface shear stress and improve particle removal. The work of Chen et al. (2009) [6] could provide insight into appropriate frequency ranges and magnitudes. Ideally a self contained nozzle design could be developed to simultaneously deliver fluid flow and acoustic radiation.

To aid in flow visualization, the impingement surface could be heated to a uniform temperature and imaged with a thermal camera. The cooling effects could be visualized as the surface is impinged by the nozzle under various flow conditions. The magnitude of heat transfer could be correlated to a magnitude of surface shear stress.

PIV measurements of current flow fields could be captured to further support computational results. The nozzle inlet could be seeded with highly reflective par- 
ticles. Flow field velocity distributions could be sampled along planes both parallel and perpendicular to the axis of rotation.

Particle removal efficiencies could be evaluated using the current nozzle design. Martian or Lunar regolith simulant could be distributed on various surfaces and then removed using bound vortex impingement. A microscope could be used to collect data on remaining particle size distributions. Experiments could be repeated using charged particles to characterize the effect of particle charge on the removal process. 


\section{References}

[1] Abbas,M. M.; Tankosic, D.; Craven, P.; Spann, J.F.; LeClair, A.; West, E.A., "Lunar dust charging by photoelectric emissions" Planet Space Sci, 2007 vol. 55 (7-8) pp. 953-965.

[2] Abrantes, J.; Fernando, L.; Azevedo, A.. "Fluid Flow Characteristics of a Swirl Jet Impinging on a Flat Plate". 13th Int Symp on Applications of Laser Techniques to Fluid Mechanics Lisbon, Portugal, 26-29 June, 2006.

[3] Ansys inc.. "FLUENT 6.3 User's Guide". Ansys inc. Fluent 6.3 support material, 2010.

[4] Benz, M., Rosenberg, K. J., Kramer, E. J., and Israelachvili, J. N., The deformation and adhesion of randomly rough and patterned surfaces, Journal of Physical Chemistry B 110(24), 11884-11893 (2006).

[5] Calle, C.I.; McFall, J.L.; Buhler, C.R.; Snyder, S.J.; Arens, E.E. ; Chen, A.; Ritz, M.L.; Clements,J.S.; Fortier, C.R. and Trigwell, S.. "Dust Particle Removal by Electrostatic and Dielectrophoretic Forces with Applications to NASA Exploration Missions" Proc. ESA Annual Meeting on Electrostatics 2008, Paper O. 
[6] Chen, Di; Wu, Junru. "Dislodgement and removal of dust-particles from a surface by a technique combining acoustic standing wave and airflow". J. Acoust. Soc. Am. 127 (1), Jan 2009.

[7] Ding, W., Zhang, H., and Cetinkaya, C., Rolling resistance moment-based adhesion characterization of microspheres, The Journal of Adhesion 84, 996-1006 (2008).

[8] Dominik, C. and Tielens, A.G.G.M., Resistance to rolling in the adhesive contact of two elastic spheres, Philosophical Magazine A 92(3), 783-803 (1995).

[9] Hirsa, A.; Lopez, J.M.; Kim, S. "Evolution of an initially columnar vortex terminating normal to a no-slip wall". Experiments in Fluids 29 pp. 309-321, 2000.

[10] Hyatt, M.; Greenberg, P.; Pines, V.; Chait, A. "Lunar and Martian Dust: Evaluation and Mitigation". 45th AIAA Aerospace Sciences Meeting and Exhibit Jan., 2007.

[11] J. Jeong and F. Hussain. "On the Identication of a Vortex". J. Fluid Mechanics, 285:69 94, 1995.

[12] Ichimiya, K.; Tsukamoto, K.. "Heat Transfer Characteristics of a Swirling Laminar Impinging Jet". Journal of Heat Transfer, Jan., Vol. 132, 2010.

[13] Kontis, K.; An, R.; Zare-Behtash, H.; Kounadis, D., "Head-on collision of shock wave induced vortices with solid and perforated walls", Physics of Fluids, Volume 20, Issue 1, pp. 016104-016104-17 (2008).

[14] Krauss,C.E.; Horanyi,M.; Robertson,S., "Experimental evidence for electrostatic discharging of dust near the surface of Mars", New J Phys, 2003 vol. 5 pp. 70. 
[15] Lu, Y.F.; Zheng, Y.W.; Song, W.D.. "An energy approach to the modelling of particle removal by pulsed laser irradiation". Appl. Phys. A 68, 569-572, 1999.

[16] Launder, B. E. and Spalding, D. B.. "Lectures in Mathematical Models of Turbulence". Academic Press, London, England, 1972.

[17] Masuda, H.; Gotoh, K; Fukada, H; Banba, Y.. "The removal of particles from flat surfaces using a high-speed air jet.", Advanced Powder Technology, Volume 5, Issue 2, 1994, Pages 205-217

[18] Mazumder, M. K.; Sharma, R.; Biris, A.S.; Zang, J., "Self-Cleaning Transparent Dust Shields for Protecting Solar Panels and Other Devices",Particulate Science and Technology, 25: p520, 2007.

[19] NASA; Carter, J., "Characterization Summery of JSC-1AF Lunar Mare Regolith Simulant.", NASA Version 1.6.2, December 12, 2006.

[20] Park, J.S.; Liu, Y.; Kihm,K.D.; Taylor, L.A., "Micro-Morphology And Toxicological Effects Of Lunar Dust". Lunar and Planetary Science XXXVII (2006).

[21] Parras, L.; Fernandez-Feria, R. "Interaction of an Unconfined Vortex with a Solid Surface". June 2007, Physics of Fluids, Vol. 19, 2007.

[22] Phillips, W.R.C.; Khoo, B.C.. "The boundary layer beneath a rankine-like vortex". Proc. R. Soc. Lond. A 1987 411, pp. 177-192.

[23] Schiller, L. and Naumann, A., ber die gundlegenden Berechungen bei der Schwerkraftaufbereitung, Ver. Deut. Ing. 77, 318 (1933).

[24] Schmeeckle, M.; Nelson, J.. "Direct numerical simulation of bedload transport using a local, dynamic boundary condition." Sedimentology, 50, pp. 279-301, 2003. 
[25] Shih, T.-H.; Liou, W. W.; Shabbir, A.; Yang, Z. and Zhu,J. ."A New - EddyViscosity Model for High Reynolds Number Turbulent Flows - Model Development and Validation". Computers Fluids, 24(3):227-238, 1995.

[26] Sweeney, L.G. and Finlay, W.H., Lift and drag forces on a sphere attached to a wall in a Blasius boundary layer. Journal of Aerosol Science 38, 131-135 (2007).

[27] Wen, M.; Jang, K.. "An inpingement cooling on a flat surface by using circular jet with longitudinal swirling strips". International Journal of Heat and Mass Transfer 46 (2003) 46574667.

[28] Zhang, XW; Yao, ZH; Hao, PF; Xu, HQ , "Study on particle removal efficiency of an impinging jet by an image-processing method", Experiments in Fluids, Volume 32, Issue 3, pp. 376-380 (2002).

[29] Ziskind, G.; Yarin, L. P.; Peles, S.; Gutfinger, C.., "Experimental Investigation of Particle Removal from Surfaces by Pulsed Air Jets. Aerosol Science and Technology", May 2002, Vol. 36 Issue 5, pp. 652-659. 


\section{Appendix A}

\section{Fluent Formulation [3]}

\section{A.1 Navier-Stokes Equations}

Continuity / Conservation of Mass:

$$
\frac{\partial \rho}{\partial t}+\nabla \cdot(\rho \vec{\nu})=S_{m}
$$

Where $S_{m}$ is the mass added to the continuous phase.

Conservation of Momentum:

$$
\frac{\partial}{\partial t}(\rho \vec{\nu})+\nabla \cdot(\rho \vec{\nu} \vec{\nu})=-\nabla p+\nabla \cdot(\overline{\bar{\tau}})+\rho \vec{g}+\vec{F}
$$

Where $p$ is the static pressure, $\overline{\bar{\tau}}$ is the stress tensor, and $\rho \vec{g}$ and $\vec{F}$ are the gravitational body force and external body forces. $\overline{\bar{\tau}}$ is defined as:

$$
\overline{\bar{\tau}}=\mu\left[\left(\nabla \vec{\nu}+\nabla \vec{\nu}^{T}\right)-\frac{2}{3} \nabla \cdot \vec{\nu} I\right]
$$

Where $\mu$ is the molecular viscosity, and $I$ is the unit tensor. 


\section{A.2 Realizable $k-\epsilon$ Turbulence Model}

Transport equations:

$$
\begin{gathered}
\frac{\partial}{\partial t}(\rho k)+\frac{\partial}{\partial x_{j}}\left(\rho k u_{j}\right)=\frac{\partial}{\partial x_{j}}\left[\left(\mu+\frac{\mu_{t}}{\sigma_{k}}\right) \frac{\partial k}{\partial x_{j}}\right]+G_{k}+G_{b}-\rho \epsilon-Y_{M}+S_{k} \\
\frac{\partial}{\partial t}(\rho \epsilon)+\frac{\partial}{\partial x_{j}}\left(\rho \epsilon u_{j}\right)=\frac{\partial}{\partial x_{j}}\left[\left(\mu+\frac{\mu_{t}}{\sigma_{\epsilon}}\right) \frac{\partial \epsilon}{\partial x_{j}}\right]+\rho C_{1} S_{\epsilon}-\rho C_{2} \frac{\epsilon^{2}}{k+\sqrt{\nu \epsilon}}+C_{1 \epsilon} \frac{\epsilon}{k} C_{3 \epsilon} G_{b}+S_{\epsilon} \\
C_{1}=\max \left[0.43, \frac{\eta}{\eta+5}\right], \eta=S \frac{k}{\epsilon}, S=\sqrt{2 S_{i j} S_{i j}}
\end{gathered}
$$

Where $G_{k}$ is the generation of turbulent kinetic energy due to mean velocity gradients. $G_{b}$ is the generation of turbulent kinetic energy due to buoyancy. $Y_{m}$ is the contribution of the fluctuating dilatation in the compressible turbulence to the overall dissipation rate. The terms $C_{2}$ and $C_{1 \epsilon}$ are constants. The terms $\sigma_{k}$ and $\sigma_{\epsilon}$ are the turbulent Prandtl numbers for $k$ and $\epsilon . S_{k}$ and $S_{\epsilon}$ are user defined source terms [3].

Turbulent Viscosity:

The eddy viscosity is computed from:

$$
\mu=\rho C_{\mu} \frac{k^{2}}{\epsilon}
$$

Where

$$
C_{\mu}=\frac{1}{A_{0}+A_{s} \frac{k U^{*}}{\epsilon}}
$$

and 


$$
U^{*}=\sqrt{S_{i j} S_{i j}+\tilde{\Omega}_{i j} \tilde{\Omega}_{i j}}
$$

and

$$
\begin{gathered}
\tilde{\Omega}_{i j}=\Omega_{i j}-2 \epsilon_{i j} \omega_{k} \\
\tilde{\Omega}_{i j}=\bar{\Omega}_{i j}-\epsilon_{i j} \omega_{k}
\end{gathered}
$$

$\bar{\Omega}_{i j}$ Is the mean rate of rotation tensor with an angular velocity of $\omega_{k}$. $A_{0}$ and $A_{s}$ are the model constants [3]:

$$
A_{0}-4.04, A_{s}=\sqrt{6} \cos \phi
$$

Where

$$
\phi=\frac{1}{3} \cos ^{-1}(\sqrt{6} W), W=\frac{S_{i j} S_{j k} S_{k i}}{\tilde{S}^{3}}, \tilde{S}=\sqrt{S_{i j} S_{i j}}, S_{i j}+\frac{1}{2}\left(\frac{\partial u_{j}}{\partial x_{i}}+\frac{\partial u_{i}}{\partial x_{j}}\right)
$$

The model constraints used in Fluent's formulation are:

$$
C_{1 \epsilon}=1.44, C_{2}=1.9, \sigma_{k}=1.0, \sigma_{\epsilon}=1.2
$$

\section{A.3 Pressure-Based Solver}

$$
\begin{gathered}
\oint \rho \vec{v} \vec{v} \cdot d \vec{A}=0 \\
\oint \rho \vec{v} \vec{v} \cdot d \vec{A}=-\oint \rho \mathbf{I} \cdot d \vec{A}+-\oint \overline{\bar{\tau}} \cdot d \vec{A}+-\oint \vec{F} d V
\end{gathered}
$$

where $\mathbf{I}$ is the identity matrix, $\overline{\bar{\tau}}$ is the stress tensor, and $\vec{F}$ is the force vector [3] . 


\section{A.4 Discretization of the Continuity Equation}

$$
\sum_{f}^{N_{\text {faces }}} J_{f} A_{f}=0
$$

where $J_{f}$ is the mass flux through face $f, \rho v_{n}[3]$.

$J_{f}$ may be written as:

$$
\left.J_{f}=\rho_{f} \frac{a_{p, c_{0}} v_{n, c_{0}}+a_{p, c_{1}} v_{n, c_{1}}}{a_{p, c_{0}}+a_{p, c_{1}}}+d_{f}\left(\left(p_{c_{0}}+(\nabla p)_{c_{0}} \cdot \overrightarrow{r_{0}}\right)-\left(p_{c_{1}}+(\nabla p)_{c_{1}} \cdot \overrightarrow{r_{1}}\right)\right)\right)=\hat{J}_{f}+d_{f}\left(p_{c_{0}}-p_{c_{1}}\right)
$$

The terms $p_{c_{0}}, p_{c_{1}}$ and $v_{n, c_{0}}, v_{n, c_{1}}$ are the pressures and normal velocities and $\hat{J}_{f}$ contains the influence of velocities in these cells. The term $d_{f}$ is a function of $\bar{a}_{p}$, which is the average of the momentum equation $a_{p}$ coefficients for the cells on either side of face $f[3]$.

\section{A.5 Pressure-Velocity Coupling}

The SIMPLE algorithm uses a relationship between velocity and pressure corrections to enforce mass conservation and to obtain the pressure field [3] .

If the momentum equation is solved with a guessed pressure field $p^{*}$, the resulting face flux, $J_{f}^{*}[3]$.

$$
J_{f}^{*}=\hat{J}_{f}^{*}+d_{f}\left(p_{c_{0}}^{*}-p_{c_{1}}^{*}\right)
$$

does not satisfy the continuity equation. A correction $J_{f}^{\prime}$ is added to the face flux $J_{f}^{*}$ so that the corrected face flux, $J_{f}[3]$.

$$
J_{f}=J_{f}^{*}+J_{f}^{\prime}
$$


satisfies the continuity equation. The $J_{f}^{\prime}$ be written as

$$
J_{f}^{\prime}=d_{f}\left(p_{c_{0}}^{\prime}-p_{c_{1}}^{\prime}\right)
$$

where $p^{\prime}$ is the cell pressure correction[3].

The SIMPLE algorithm substitutes the flux correction equations into the discrete continuity equation to obtain a discrete equation for the pressure correction $p^{\prime}$ in the cell [3]:

$$
a_{P} p^{\prime}=\sum_{n b} a_{n b} p_{n b}^{\prime}+b
$$

where the source term $b$ is the net flow rate into the cell:

$$
b=\sum_{f}^{N_{f} \text { aces }} J_{f}^{*} A_{f}
$$

Once a solution is obtained, the cell pressure and the face flux are corrected using

$$
\begin{gathered}
p=p^{*}+\alpha_{p} p^{\prime} \\
J_{f}=J_{f}^{*}+d_{f}\left(p_{c_{0}}^{\prime}-p_{c_{1}}^{\prime}\right)
\end{gathered}
$$

Here $\alpha_{p}$ is the under-relaxation factor for pressure. $J_{f}$ is the corrected face flux [3] .

\section{A.6 Second-Order Upwind Scheme}

The face value $\phi_{f}$ is computed using the following expression [3] :

$$
\phi_{f, S O U}=\phi+\nabla \phi \cdot \vec{r}
$$


Where $\phi$ and $\nabla \phi$ are the cell-centered value and its gradient in the upstream cell. $\vec{r}$ is the displacement vector from the upstream cell centroid to the face centroid. The gradient $\nabla \phi$ is determined in each cell. [3] . 


\section{A.7 Nozzle Design}

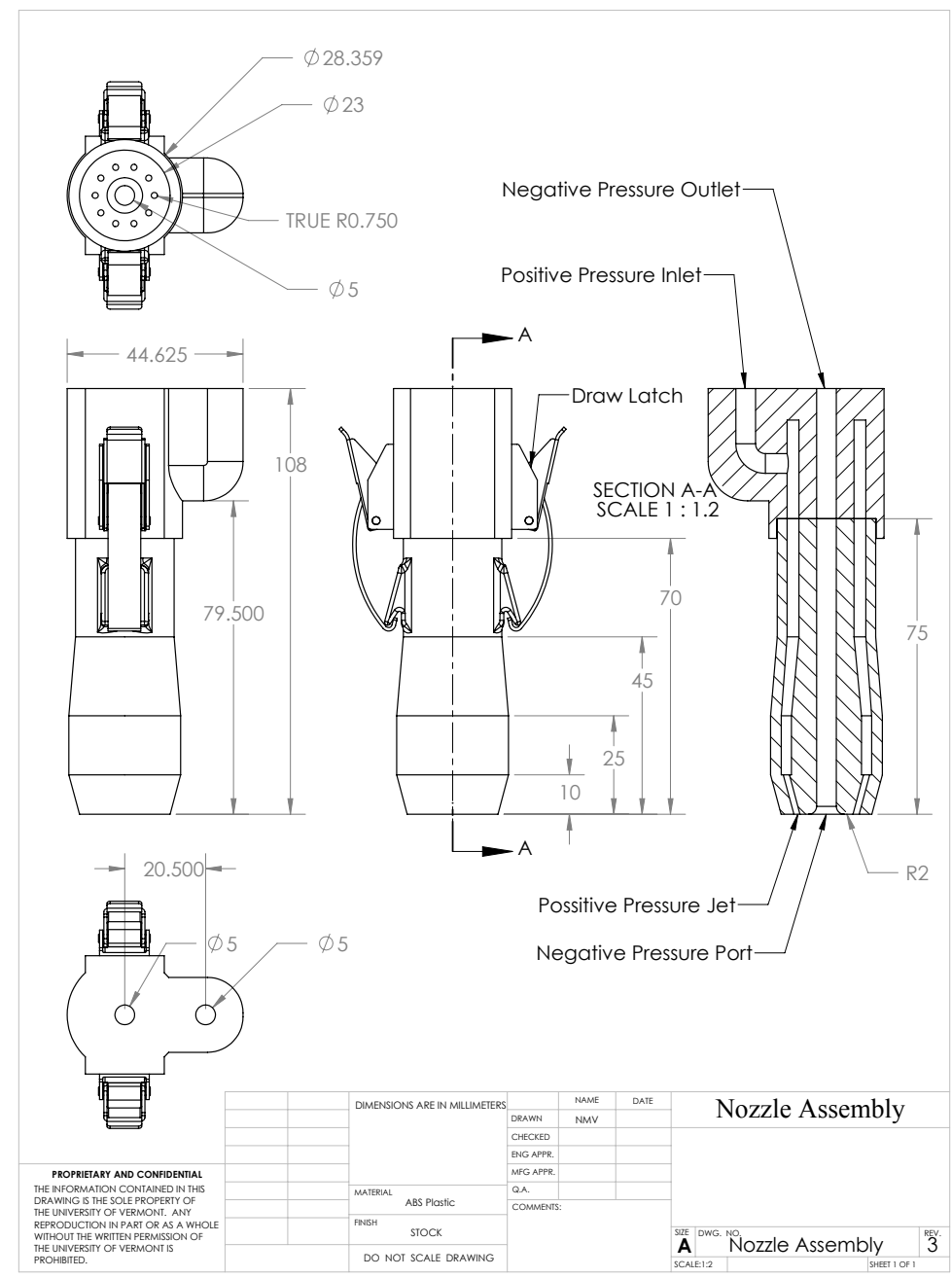

Figure A.1: A technical drawing of the experimental nozzle assembly is shown. The lower portion of the nozzle can be detached and exchanged with an alternative tangential and radial configuration. A $0^{\circ}$ tangential component and $15^{\circ}$ radial component is depicted. 


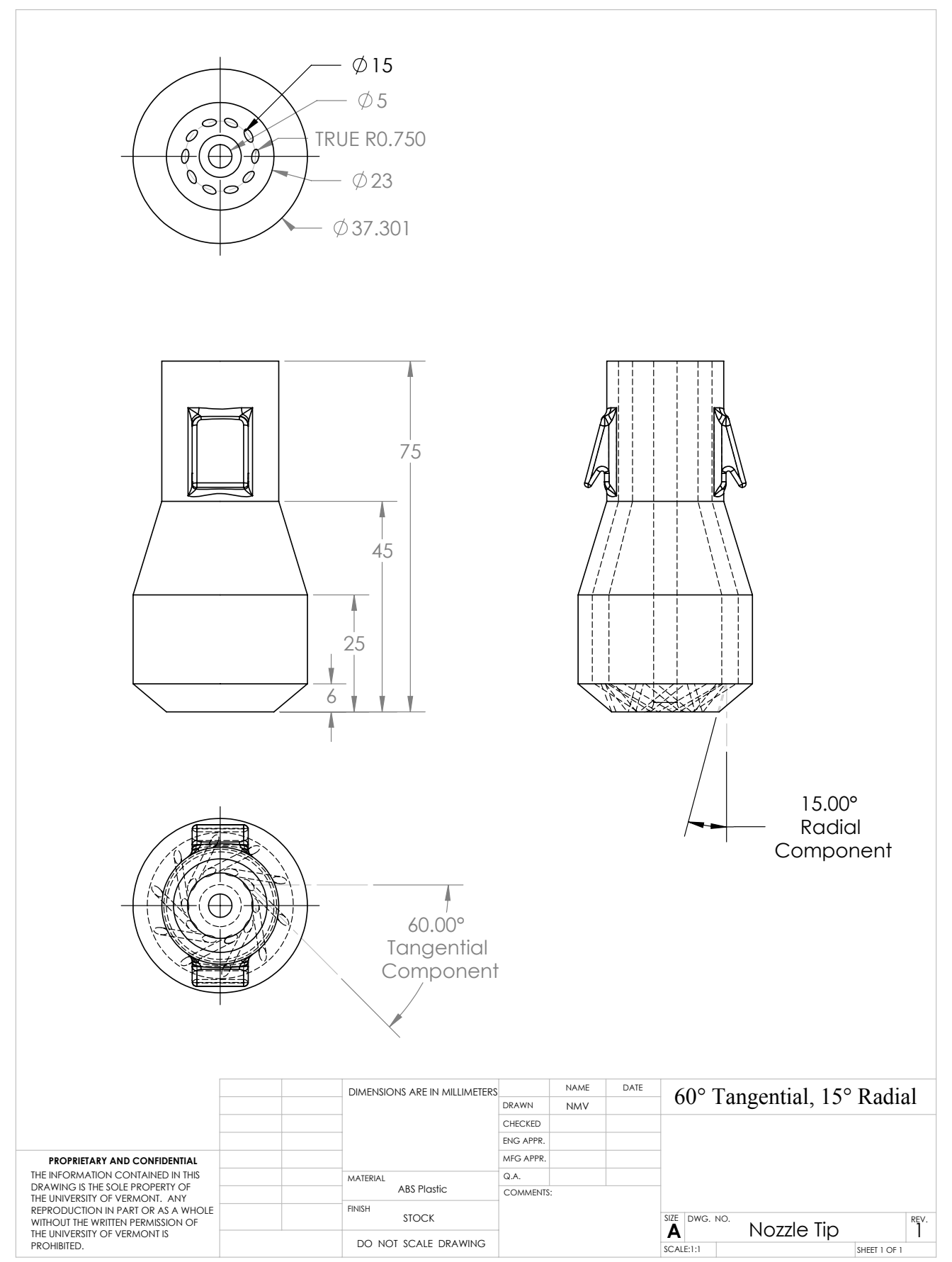

Figure A.2: A technical drawing of the 'optimal' experimental nozzle is shown. A $60^{\circ}$ tangential component and $15^{\circ}$ radial component is depicted. 